\title{
A roadmap for the clinical implementation of optical-imaging biomarkers
}

Dale J. Waterhouse ${ }^{1,2}$, Catherine R. M. Fitzpatrick ${ }^{2,3}$, Brian W. Pogue ${ }^{4}$, James P. B. O'Connor ${ }^{5}$ and Sarah E. Bohndiek $^{1,2^{\star}}$

${ }^{1}$ Department of Physics, University of Cambridge, United Kingdom, ${ }^{2}$ Cancer Research UK Cambridge Institute, University of Cambridge, United Kingdom, ${ }^{3}$ Department of Engineering, University of Cambridge, United Kingdom, ${ }^{4}$ Thayer School of Engineering, Dartmouth, USA, ${ }^{5}$ Division of Cancer Sciences, University of Manchester, United Kingdom.

*Department of Physics, University of Cambridge, Cavendish Laboratory, JJ Thomson Avenue, Cambridge, CB3 OHE, United Kingdom, and Cancer Research UK Cambridge Institute, University of Cambridge, Li Ka Shing Centre, Robinson Way, Cambridge CB2 0RE, United Kingdom. e-mail: seb53@cam.ac.uk

Clinical workflows for the non-invasive detection and characterization of disease states could benefit from optical-imaging biomarkers. In this Perspective, we discuss opportunities and challenges towards the clinical implementation of optical-imaging biomarkers for the early detection of cancer by analysing two case studies: the assessment of skin lesions in primary care, and the surveillance of patients with Barrett's oesophagus in specialist care. We stress the importance of technical and biological validations and clinical-utility assessments, and the need to address implementation bottlenecks. In addition, we define a translational roadmap for the widespread clinical implementation of optical imaging-technologies.

Optical-imaging biomarkers (OIBs), which rely on the interactions of tissue and non-ionizing optical radiation (with typical wavelengths in the range of $400-1,000 \mathrm{~nm}$ ), can be used for the non-invasive detection and characterization of disease states. OIBs enable the real-time analysis of tissue biochemistry and the use of compact point-of-care and low-cost imaging devices (when compared to radiological imaging), and can operate across ranges of resolutions and depths spanning over four orders of magnitude ${ }^{1}$.

Across the visible and near-infrared spectrum, light undergoes a range of complex interactions with tissue (Fig. 1). Conventional photographic methods that aim at replicating human vision ${ }^{2}$ discard most of the information obtained from these interactions and only capture reflected light across three channels (red, green and blue). Over the past decade, a wide range of promising OIBs that extract in-depth information provided by the different light-tissue interactions have emerged. However, for any new imaging biomarker to be deployed in a clinical setting, detailed validation is required. Technical validation defines the precision and accuracy with which the biomarker can be measured, whereas biological validation establishes the association between the biomarker and the underlying physiological, anatomical or pathological process. Clinical validation can then establish whether the biomarker does indeed identify, measure or predict the clinical outcome of interest. To achieve clinical validation, the imaging device needs to conform to clinical performance and safety specifications, and be approved for use in patients.

With standard radiological imaging — such as computed tomography (CT) or magnetic resonance imaging (MRI) - the imaging device required to measure a novel imaging biomarker is already clinically approved for use in humans and widely available across radiology departments ${ }^{3}$. In contrast, for OIBs it is uncommon that a clinically approved imaging device (alongside its associated specialist data-acquisition and data-interpretation methods) is available for clinical validation. Therefore, biological validation may be restricted to testing ex vivo samples such as histopathological sections. Compared to the in vivo setting, these can be prone to bias, and generate a different range of optical interactions, ultimately resulting in misleading conclusions as to the potential clinical utility of the OIB (ref. ${ }^{4,5}$ ). Furthermore, OIBs may be deployed in a range of settings during the patient-management pathway, spanning primary care (such as a family physician) and specialist care (specialist practice, referral, or medical centre). Hence, even a welldefined OIB with promising performance in an experimental setting may not receive approval if it fails to adequately address the specific diagnostic question at a defined point in the patient-management pathway.

Given the promise of emerging OIBs, in this Perspective we discuss challenges and opportunities for their clinical translation, from development to implementation in healthcare systems. To this end, we selected two distinct case studies in early cancer detection, and identified a number of OIBs that have reached maturity in clinical use (Table 1). The first case study covers primary care in the context of the assessment of melanocytic skin lesions, and the second case study covers specialist care in the context of the surveillance of patients with Barrett's oesophagus. We use trends identified from both cases to define a set of translational characteristics that contribute to the likelihood of a new OIB being incorporated into healthcare, accelerating translation during both technical and biological validation through to the assessment of clinical utility. Taking a page from the recent consensus roadmap for radiological imaging biomarkers ${ }^{3}$, we propose an OIB roadmap that links the translational characteristics of OIBs and defines the key bottlenecks that must be overcome in order to facilitate a smoother path for their clinical translation.

\section{Early detection of melanoma in primary care}

Although malignant melanoma represents less than $5 \%$ of cutaneous malignancies, it causes the majority of skin-cancer deaths $^{6}$. In the United Kingdom, the current management of patients presenting with a pigmented skin lesion involves a primary-care general practitioner assessing the patient's history and performing a naked-eye visual inspection of the lesion, guided by a seven-point checklist: lesion size (diameter larger than $7 \mathrm{~mm}$ ), change in lesion size, and the presence of irregular pigmentation, of an irregular border, of inflammation, of itch or altered sensation, and of oozing or crusting of the lesion. If the lesion appears suspicious on the basis of this visual assessment, the patient is referred to specialist care via an urgent skin-cancer pathway ${ }^{7}$. 
The potential benefit that population-screening programmes could have in malignant melanoma management is evidenced by the improved survival of patients for whom melanomas are detected by physicians rather than by patients or family members ${ }^{8}$. This occurs because lesion thickness is a key determinant of patient outcome (the five-year survival rate improves to $91 \%$ for lesions smaller than $1-\mathrm{mm}$ thick from a mere $46 \%$ for lesions larger than $4-\mathrm{mm}$ thick ${ }^{9}$ ), and because physicians can typically recognize thinner lesions. A large population study suggested that an almost $50 \%$ reduction in mortality rates is possible by adopting full-body skin examinations performed by dermatologists ${ }^{10,11}$. However, owing to methodological limitations of the study ${ }^{12}$, it did not provide sufficient evidence to recommend a national population screening programme.

Because the skin provides an easily accessible surface for optical imaging, OIBs can improve the triage of suspected malignant melanoma in primary care, and provide sensitivity and specificity for diagnosis in specialist care, thus avoiding unnecessary biopsies when possible. Optical-imaging approaches can be broadly categorized as methods that enable the visualization of the tissue with spatial resolution (imaging), and methods that map point-based biochemical information (spectroscopy). A few multispectral-imaging techniques are, however, capable of combining both types of information. In what follows, we review devices that have received regulatory approval for use in humans in each of these categories.

Imaging. Dermatologists use a handheld dermoscope (a magnifying optical-imaging instrument) in about $78 \%$ of examinations of suspected malignant melanoma (ref. ${ }^{13}$ ). According to a meta-analysis of 8,487 suspicious skin lesions, a dermoscope improves the detection sensitivity from $71 \%$ to $90 \%$ and the specificity from $81 \%$ to $90 \%$ (ref. ${ }^{14}$ ) with respect to examination solely by the naked eye. Multispectral-imaging methods employ handheld devices to illuminate the tissue with broadband white light, and measure the reflected light at several different wavelengths (typically, up to ten). The resulting spectral images are then processed, often using reference spectral properties of prominent tissue absorbers, such as melanin, haemoglobin, water and lipids. For example, SIAscopy (MedX, Canada) measures the reflected light of the skin at eight different wavelengths. The data are then used to generate maps of melanin, dermal melanin, haemoglobin and collagen in the skin using model-based fitting ${ }^{15}$. The MoleMate system (MedX, Canada) combines SIAscopy with a primary-care scoring algorithm. The algorithm interprets these maps and classifies lesions as 'suspicious', 'not suspicious', 'haemangioma', or 'seborrheic keratosis'16. A large primary-care randomized controlled trial $^{17}$ of the MoleMate system in the United Kingdom showed that incorporating the use of the device into existing bestpractice clinical guidelines (including the seven-point checklist) led to similar diagnostic accuracy for 'suspicious' lesions as that of current practice ${ }^{18}$. Furthermore, clinicians and patients rated the device more highly for 'reassuring and thorough care'18. However, as its use resulted in more referrals from primary care, it was not recommended for use in routine primary care.

Two additional multispectral-imaging methods have been examined for the inspection of malignant melanoma. SkinSpect (Spectral Molecular Imaging, USA) produces melanin and haemoglobin maps similar to those of MoleMate ${ }^{19-22}$ also using model-based fitting, so it may be possible to include SkinSpect into established scoring algorithms. MelaFind (Strata Skin Science, USA; formerly Mela Science), which has been discontinued, took a different approach. Instead of using automatic segmentation, it performs feature extraction and classification ${ }^{23}$ to translate the multispectral-imaging data into a binary biomarker classification: 'biopsy' or 'no-biopsy'24. In a study comparing German and United States dermatologists, German dermatologists were less likely to incorporate MelaFind into their decision-making. It was suggested that the German physicians' older age, more extensive dermoscopy training, and unfamiliarity with MelaFind (ref. ${ }^{25}$ ) contributed to the less-frequent use of the technology, thus indicating that MelaFind may find a niche in assisting less-experienced dermatologists or those without training in dermoscopy. Despite the widespread availability of multispectral-imaging methods for the assessment of suspected malignant melanoma, none of these methods have been incorporated into routine use or into decision-making algorithms.

Reflectance confocal microscopy (RCM) captures high-resolution images of the epidermis and papillary dermis with almost cellular resolution ${ }^{26}$. These images are interpreted via a feature-based scoring algorithm that classifies a binary biomarker: 'non-MM' and 'MM'(ref. ${ }^{27}$ ). RCM has been implemented in the VivaScope imaging systems (Caliber I.D., USA), which were found to significantly increase specificity when compared to dermoscopy in a recent meta-analysis ${ }^{26}$ of eight studies ${ }^{28-35}$. However, national authorities such as NICE in the UK do not recommend its use because of insufficient evidence of a clinically relevant advance ${ }^{36}$. High-resolution images can also be obtained with Dermalnspect (Jenlab, Germany), a multiphoton-tomography-fluorescence-lifetime-imaging device that measures time-resolved tissue autofluorescence ${ }^{37,38}$ to detect metabolic and structural molecules in tissue. In comparison to simple handheld devices conventionally used by dermatologists, the device is much larger and more expensive. Preliminary results for diagnosing malignant melanoma using Dermalnspect are promising ${ }^{39}$, but further studies in larger patient cohorts are needed to evaluate whether Dermalnspect can improve the sensitivity or specificity for the diagnosis of malignant melanoma.

Optical coherence tomography (OCT) captures high-resolution cross-sectional images of the skin by a method that is similar to ultrasound ${ }^{40}$. Compared to dermoscopy or confocal techniques, OCT enables the imaging of skin layers deeper than $1 \mathrm{~mm}$. Pilot studies have demonstrated the potential of OCT for the detection of malignant melanoma ${ }^{41,42}$. Two devices are currently in clinical use with similar technical specifications ${ }^{43}$ : Skintell (Agfa Healthcare, Belgium and Germany) and Vivosight (Michelson Diagnostics, UK). Since cross-sectional OCT images are similar to histological images, and en-face OCT images are similar to confocal-microscopy images, OCT is able to use feature descriptors from pathologists, including architectural patterns and cytological features of pigmented cells in the epidermis, dermoepidermal junction and dermis, providing a starting point for feature-based algorithms for disease classification and biomarker development ${ }^{41}$. The widespread adoption of OCT in ophthalmology ${ }^{44,45}$ may contribute to the successful application of OCT in the diagnosis of malignant melanoma, but additional clinical trials are needed to evaluate whether there is sufficient added value in depth-resolved OCT images. 
Spectroscopy. In spectroscopy methods, the tissue is illuminated, and changes in wavelength or intensity that result from light absorption, light scattering or fluorescence interactions of light and tissue ${ }^{46,47}$ are measured. These methods differentiate malignant from benign tissue on the basis of the properties of the bulk chemical constituents of tissue such as water, lipids, proteins, RNA, DNA, haemoglobin and melanin - or of specific structural (in particular, collagen) or metabolic (such as NADH) molecules ${ }^{46,47}$. Spectroscopy-based modalities are typically limited to a narrow field of view or to even mapping with a single point, yet acquire data at high spectral resolution (often measuring the intensity at hundreds or thousands of different wavelengths). The high dimensionality of spectral data prohibits interpretation by the human eye, so this data is usually analysed via multivariate statistical techniques, or increasingly by machine-learning approaches, to reach a binary biomarker classification, with histopathology providing the ground truth for training these algorithms ${ }^{48}$. Perhaps owing to the complexity of the data, most spectroscopy methods remain firmly in the preclinical phase and have not achieved regulatory approval ${ }^{49,50,59,51-58}$.

Raman spectroscopy is one optical-spectroscopy modality that has received regulatory approval. It probes the primary chemical constituents of tissue via inelastic scattering. The acquired spectra are classified using principal component analysis and discriminant analysis, both based on a training set of known spectra for which the classifications are known a priori. Several large clinical studies have demonstrated the potential of Raman spectroscopy in skin imaging. One of these studies ${ }^{55}$ has resulted in a commercial clinical system (Aura; Verisante, Canada; ref. ${ }^{61}$ ) that has received regulatory approval. Nonetheless, despite over two decades of research, Raman spectroscopy is not routinely used in the clinic for the evaluation of malignant melanoma. The slow uptake might be due to challenges in obtaining an accurate ground truth for training the classifiers ${ }^{60,61}$, and to challenges of quality assurance and control in spectral calibration and spectra interpretation ${ }^{48}$. Recent developments have significantly shortened the signal acquisition times $(<1 \mathrm{~s}$ in endoscopy), which may solve some of these problems by allowing faster and more robust signal acquisition from tissues that are inherently heterogeneous, allowing for better delineation of the rich spectral features enabled by this imaging modality.

Considerations for technology adoption in primary care. One key to improving the outcomes of malignant melanoma will be earlier disease detection. To achieve this, detection methods have to minimize false-negative rates and be easily implementable while maintaining a high negative predictive value. Afterwards, cost-effectiveness can be considered, via an evaluation of the entire impact of the technology with regards to complete patient management.

Another matter is whether primary-care physicians are willing to use new imaging methods. Although there is little doubt as to whether microscopic tools such as RCM provide better information than macroscopic imaging systems (and then naked-eye examination), the added time required to image entire macroscopic fields of view, or to choose which microscopic fields to image, is a challenge for the adoption of imaging technology in primary care. Methods for automated mosaicking with microscopy hold substantial promise to improve this, but the logistical problem of mapping out a macroscopic field of view with a microscopic tool is one that may need to be solved in order to facilitate wide adoption of the technology.

\section{Early detection of dysplasia in Barrett's oesophagus}

Barrett's Oesophagus (BO) is an acquired condition in which columnar epithelium replaces the stratified squamous epithelium of the distal oesophagus. Patients with BO have an elevated risk of developing oesophageal adenocarcinoma ${ }^{62,63}$, which increases in the presence of dysplasia ${ }^{64,65}$. As a result, patients with BO are often recommended to undergo routine surveillance with high-definition white light endoscopy (HD-WLE) on a three-to-five year basis ${ }^{66-70}$. Targeted and random biopsies are taken during endoscopy, and analysed by a pathologist for the identification of cellular changes associated with dysplasia.

Although several studies have shown that a surveillance regime detects cancers at an earlier stage and increases survival ${ }^{71-75}$, its sensitivity remains as low as $40 \%$ (ref. ${ }^{76}$ ), thus resulting in a high rate of missed dysplastic lesions ${ }^{77}$. The main reasons for the low sensitivity are that dysplasia can be difficult to spot on HD-WLE (ref. ${ }^{67}$ ) and that taking random biopsies is time consuming, costly, and prone to sampling errors ${ }^{67}$. New developments in optical imaging for addressing these limitations fall into two main categories: advances in wide-field imaging aim to provide better visualisation of dysplasia and to provide a 'red flag' for the endoscopist to target the biopsy; and advances in narrow-field imaging provide an 'optical biopsy' of the suspicious areas that could ultimately reduce or replace tissue biopsy. The combination of a high sensitivity 'red flag' approach with a high specificity 'optical biopsy' approach may prove to be a useful strategy in endoscopic imaging for BO (and for other cancers in the Gl tract). According to the guidelines of the American Society for Gastrointestinal Endoscopy (ASGE), a new technology needs to have $90 \%$ sensitivity, $80 \%$ specificity and $98 \%$ negative predictive value to be recommended for targeted biopsy ${ }^{78}$. To date, only three technologies have met these thresholds: narrow band imaging (NBI), acetic acid chromoendoscopy and endoscope-based confocal laser endomicroscopy (eCLE).

Wide-field imaging. In chromoendoscopy, dyes are applied to enhance the contrast in tissues so as to improve the detection of early lesions. The most common dyes are methylene blue ${ }^{79,80}$ (a vital stain that is absorbed by the tissue) and acetic acid $77,81,82$ (a weak acid that causes a transient whitening of the tissue and accentuates the mucosal pit pattern). The optical biomarker in these cases is the presence or absence of a pattern of dye staining, which is normally agreed by expert consensus. Although chromoendoscopy helps to visualise the tissue structure without the need for specialized equipment, the application of the dye requires additional tissue preparation, adding time and costs to the procedure, which is not widely reimbursed 5 . Chromoendoscopy using acetic acid has been clinically recommended to increase the yield of targeted biopsy ${ }^{5}$, but for methylene blue phototoxicity concerns remain ${ }^{83-85}$. Numerous research groups are developing targeted optical molecular-imaging dyes that enable the visualisation of complex biochemical processes involved in disease ${ }^{86,87}$. These dyes bind disease-specific cell-surface receptors or detect other disease- 
specific molecular changes, revealing particular pathologies as regions of altered fluorescence. As with traditional chromoendoscopy, the use of these dyes would increase the time and cost of an endoscopy procedure; furthermore, the cost of synthesising new targeted dyes at clinical grade and of performing clinical trials to prove efficacy can be prohibitive. Thus, despite promising results ${ }^{88-91}$, a convincing demonstration of a significant improvement in clinical performance is still required to justify the added complexity of using optical molecular imaging in routine endoscopic surveillance.

To avoid the need for dyes, virtual (or electronic) chromoendoscopy (VC) can be employed to enhance the contrast by illuminating the tissue via restricted bandwidths of light, as in narrow-band imaging (NBI, Olympus) and blue-laser imaging (BLI, Fuji $)^{92,93}$. Alternatively, post-processing software can be used to enhance the images, as in intelligent colour enhancement (FICE, Fuij) $)^{94}$ and iSCAN (Pentax) ${ }^{95}$. Post-processing software has been shown to be equivalent or superior to HD-WLE or NBI in several gastrointestinal indications ${ }^{96-105}$, with reasonable inter-observer agreement ${ }^{106,107}$. VC requires only minor hardware or software modifications to the standard endoscopy equipment and does not require any dye that could create toxicology concerns. Hence, VC has received regulatory approval and is widely available. NBI using a feature-based classification ${ }^{108}$ has also met the ASGE threshold for clinical recommendation in targeting biopsy ${ }^{5}$.

Exploiting a different contrast mechanism, autofluorescence imaging (AFI) relies on the intrinsic fluorescence emission of structural (such as collagen) and metabolic (such as NADH) molecules in tissue ${ }^{109}$ to detect dysplasia as dark purple patches of reduced AFI signal ${ }^{110}$. Although AFI initially showed promising results, its low specificity ${ }^{110}$ prevented it from replacing WLE in the clinic. Instead, AFI has been combined with HD-WLE and VC in standard commercial forwardfacing endoscopes to create trimodal imaging systems ${ }^{111-113}$.

Endomicroscopy and spectroscopy. Once suspicious areas have been identified via wide-field imaging, a tissue biopsy is taken for histopathological analysis. Given the time and cost required for pathological assessments, several high-resolution endoscopy (or endomicroscopy) modalities have been developed with the goal of replacing tissue biopsies with in situ optical biopsies. In these approaches, high-resolution images of the epithelium are captured and interpreted using a set of consensus-feature-based criteria to classify pathological states ${ }^{144,115}$. Usually, an exogenous contrast agent, such as fluorescein, is added to enhance the contrast.

Owing to the need for confocal laser excitation and image collection optics, confocal laser endomicroscopy (CLE) is performed over a narrow field of view by introducing a probe into the accessory channel of a standard endoscopy (probebased, pCLE) or by replacing the standard endoscope with a standalone endomicroscope (eCLE). pCLE has the advantage of being compatible to the standard endoscopy procedure and has received regulatory approval (Cellvizio, Mauna Kea). eCLE (FIVE 1, Optiscan; ISC 1000, Pentax) has also received regulatory approval and meets the standards for use in targeting biopsy, but is no longer commercially available.

Volumetric optical coherence tomography (VOCT, also known as volumetric laser endomicroscopy, VLE) is an alternative endomicroscopy technique able to capture high-resolution depth-resolved cross-sectional images of the entire distal oesophagus ${ }^{116-118}$ through a balloon-based system (NVisionVLE, Ninepoint Medical) ${ }^{119-123}$. As with pCLE, the balloon is introduced via the working channel of a standard endoscope. VOCT images have been shown to correlate with histological image ${ }^{124-126}$ and can be interpreted by a trained image interpreter using a set of feature-based criteria to perform disease classification ${ }^{127}$. However, the dissimilarity between cross-sectional OCT images and standard HD-WLE image limits their co-registration and makes it challenging for endoscopists to re-locate suspicious areas identified on OCT for WLE-guided intervention. Cautery marking is currently being investigated to solve this problem ${ }^{128}$. A further consequence of the lack of familiarity of the cross-sectional OCT images is the need for advanced training of expert image interpreters, although it is possible for these experts to identify dysplasia ${ }^{129}$ with good inter-observer agreement ${ }^{127}$. Another barrier that limits widespread translation is that manual image analysis takes several hours. In this regard, automated segmentation algorithms may help ${ }^{130}$. Automated algorithms may also be combined with the emerging tethered capsule OCT endoscopy systems ${ }^{117}$ that can be swallowed un-sedated under the supervision of a nurse in primary care ${ }^{131}$. As cross-sectional information is particularly important in the assessment of submucosal invasions when staging oesophageal adenocarcinoma, further developments to improve imaging at depth, for example, by using photoacoustic imaging ${ }^{132}$, are still needed.

In addition to endomicroscopy imaging, Raman spectroscopy has been applied endoscopically to obtain biochemical information of the tissue so as to distinguish dysplasia from surrounding Barrett's tissue on the basis of least-squares discriminant analysis of the Raman spectra. But low reproducibility, expensive equipment, and the need for long integration times, have limited clinical translation. However, recent improvements in integration times ${ }^{133,134}$ and in classification algorithms to provide the endoscopist with auditory feedback of a diagnosis in real time (0.2 seconds) led to in vivo trials ${ }^{133}$. Larger-scale in vivo trials and calibration methods will be needed to improve reproducibility and to ensure that promising ex vivo results apply in vivo 60.

Considerations for technology adoption in specialist care. In primary care the consequences of false negatives are particularly acute, since no further follow up will be provided to the patient. In specialist care, however, the number of patients is lower than in primary care, and the trade-off between acceptable levels of false-positive and false-negative results depends on the next step of disease management. Ideally, both should be minimized. As such, the cutting edge of new technology is often found in specialist care, where novel techniques could add value to the decision-making process. Defining the value added of a given technology requires rigorous testing for diagnostic accuracy, acceptability, effectiveness and cost-effectiveness in the intended setting, by using case-controlled, randomized, and double-blinded clinical trials so as to assess the technology's real value to the healthcare system. If the diagnosis is more accurate or the amount of time a specialist is required is reduced, then the technology may add value. Regulatory approvals for devices, particularly with predicates, often lack clinical evidence from such trials ${ }^{135}$, so it is common for approved technologies to fail to achieve widespread adoption, because they do not change clinical practice. [Recent changes] [https://eur-lex.europa.eu/legal-content/ENG/TXT/PDF/?uri=CELEX:32017R0745] to the EU medical device legislative 
framework may mitigate this challenge in the future. Thus, the development pipeline of optical imaging devices and their associated OIBs continues well beyond regulatory approval. Most commonly, consensus studies from specialist societies are required to reach the recommendation for use in routine patient management.

\section{Translational characteristics of successful OIBs}

The two case studies discussed reveal a wide range of promising optical methods that have been established in a research setting and that could potentially improve early cancer detection. However, few of these methods have successfully received regulatory approval, and even fewer have progressed to recommendation in healthcare systems.

Cost-effectiveness is a key consideration for adoption into healthcare systems, and it applies to one-off costs (such as the purchase of instrumentation and the training of operators and interpreters) and to ongoing costs (such as the employment of operators, image interpreters and support staff, the purchase of disposable materials, cleaning or sterilization, and annual maintenance). In private healthcare systems, the development of new reimbursement codes can promote translation, as it has been the case with OCT in the context of opthalmology ${ }^{136}$. Ultimately, acceptable costs would be determined on the basis of the overall performance of the OIB and whether it increases quality-adjusted life years (QALYs) or it reduces other healthcare costs. Examples of healthcare costs that could be alleviated with successful translation of a new OIB for early cancer detection include those costs associated with treating more advanced disease (approximately a further $£ 10,000$ per patient for late-stage colorectal cancer with respect to patients with the early stage of the disease ${ }^{137}$ ) or those associated with exhaustive physical biopsies (for example, targeting biopsies in BO surveillance can reduce per-patient biopsy costs in the UK from approximately $£ 1,000$ to $£ 30$; ref. ${ }^{77}$; such costs considerably depend on country). Assessments of OIB cost-effectiveness should therefore be made in light of the intended diagnostic indication and of the potential change in the current clinical patient pathway that could be achieved.

Having achieved cost-effectiveness, OIBs still face a wide range of hurdles in clinical translation. In reviewing the two case studies, we identified several translational characteristics that are common among widely-adopted OIBs. Most of the identified translational characteristics fall into three main categories: device and methodology, image acquisition and handling, and image interpretation. These separate technical aspects of the optical-imaging modality (detailed below together with suggestions for how to best achieve clinical translation) from biomarker measurements and image analysis.

Contrast mechanism underlying the OIB. Exploiting endogenous contrast (imaging without application of dyes) to detect the OIB is favourable for a clinical application, although exogenous contrast agents can improve the contrast of cancer tissue compared to healthy tissue, both as non-specific stains ${ }^{138}$ and as targeted molecular-imaging agents ${ }^{86}$. However, contrast agents require synthesis with good manufacturing practice (GMP) standards, toxicology studies, specific instrumentation, and additional procedure time and $\operatorname{cost}^{76,139}$. Several aspects of contrast-agent chemistry increase the likelihood of their successful clinical translation: having a validated target (structural or molecular) increases confidence in the reproducibility of the results; administering agents topically rather than intravenously limits agent exposure to the tissue of interest and speeds up procedures; and using formulations of agents with long-term stability is favourable.

Instrumentation for OIB measurement. If a new device is required to measure the OIB, the device must be approved by regulatory bodies such as the FDA (refs. ${ }^{135,140-142}$ ). For clinical implementation, compact, robust and transportable optical-imaging devices are highly desirable and more likely to succeed in clinical translation, while complex or delicate optics are less amenable to clinical implementation. Furthermore, devices that are compatible with existing systems, or that include current standard-of-care methods for reference, are more likely to reach deployment in healthcare systems, as they do not require a complete overhaul of existing equipment and facilities. The compatibility with current systems also facilitates head-to-head trials and allows a modality to be introduced to the clinic as an adjunct to an existing modality. Compatibility with the existing clinical workflow, and the avoidance of major changes to procedure times or costs, are additional advantages.

Device operator expertise. The potential operators of optical instruments for measuring OIBs may be lay people, professionals working in primary care, or highly specialized individuals working in a specialist care centre. If a new approach gives similar clinical results to an existing approach, yet requires less expertise, the likelihood of clinical translation is higher. Furthermore, if the need for training is sufficiently reduced, the approach may be translated from an expert to a generalist setting, reducing the burden on specialist care centres and the cost of running a high-volume imaging suite ${ }^{117}$. Conversely, if a high level of specialist knowledge is required to measure an OIB compared to the existing standard-of-care, the OIB is unlikely to be widely adopted, unless evidence can be provided that it contributes to improved clinical outcomes. Excessive complexity in the name of additional device performance may deter clinical adoption ${ }^{143}$. Clear standard operating procedures should be determined and adhered to improve 'ease of use' for operators and the increase likelihood of translation.

Some devices may be clinically translated because they reassure patients and clinicians of improved outcomes, whether or not they actually improve the outcome. One example is the MoleMate skin-imaging system, which despite not being more accurate than best practice for detecting melanoma, it reassured patients and clinicians that they were receiving or providing thorough care ${ }^{18}$.

OIB interpreter expertise. Converting the raw-image data acquired using the optical modality into clinically relevant OIB information involves establishing image-interpretation criteria needed to deliver high sensitivity, specificity, interinterpreter agreement and short training times. Criteria can include a binary decision, a library-based classification, the presence of specific image patterns ${ }^{108,144}$, or a change in signal intensity relative to a defined threshold. Several caveats can complicate this process. For example, increased expertise does not necessarily imply better inter-interpreter agreement ${ }^{145,146}$, and when applied to videos ${ }^{147}$ the criteria often result in lower performance than originally reported in 
still-image interpretation ${ }^{148-150}$. Establishing these OIBs is a time-consuming task, often requiring international consensus across multiple centres after regulatory approval of a device. Determining their clinical performance then requires randomized controlled trials in the appropriate settings, which are expensive and difficult to implement in a diagnostic setting for screening and surveillance programmes. For more complex biomarkers, expert image interpreters need to be trained, which further adds to the cost and time for adoption and makes the biomarker difficult to standardize across centres. Simplifying the output of the biomarker or transforming it to familiar images reduces the need for retraining, may enable non-experts to make diagnoses, and may smooth the translational pathway.

Automated analysis that provides clear feedback to the interpreter of the biomarker can minimize the need for expert image interpreters. For many optical-imaging approaches, data reduction is essential because the dimensionality of the data is beyond interpretation by the human image interpreter. It has the potential to be objective, standardized and cheaper than employing human expertise, but has yet to mature to a stage where it is fully capable of operating in real time with sufficient performance to replace the human image interpreter ${ }^{151,152}$. If the technology is to be adopted in primary care, it will be important to use the device in a way that provides expert-level diagnostics with a high negative predictive value.

Repeatability and reproducibility of OIB measurements. Both repeatability and reproducibility across devices, operators and image interpreters must be assessed to evaluate the achievable precision for the measurement of the OIB. Although inter-analyst agreement (encompassing operators and image interpreters) is often assessed, intra-analyst, intra-device, and inter-device variabilities are often overlooked, making the comparative evaluation of OIBs challenging. To maximize the opportunity for translation to the intended setting, studies should be designed to enable the comparison of results obtained across multiple centres.

Co-registration of OIB information. The spatially resolved information obtained via imaging is often useful during a later surgical procedure for guiding tumour resection. However, optical-imaging modalities do not always provide sufficient anatomical information to guide an intervention. Strategies to overcome this (with increasing levels of complexity that could hinder translation) are co-registration with an existing modality that is compatible with surgical treatment $^{153}$, application of laser cautery marks to highlight target areas ${ }^{128}$, and projection of the image data onto the patient or into the surgeon's field of view via augmented-reality technology ${ }^{154,155}$.

The aforementioned wide-field endoscopy techniques produce 2D images that are easily integrated into standard equipment for HD-WLE, facilitating the widespread uptake of OIBs in endoscopy (compared to dermoscopy-based approaches $66,111,113,156)$. Acetic acid chromoendoscopy and NBI were recently reported to meet the thresholds required for recommendation ${ }^{5}$, and this milestone was achieved in part because of their deployment at many sites internationally, enabling the extensive development of image-classification criteria and publication of consensus statements ${ }^{108,148-}$ $150,157,158$

\section{A roadmap for OIBs}

We have formulated an OIB roadmap (Fig. 2), adapted from the international consensus 'Imaging Biomarker Roadmap' created for use in cancer studies ${ }^{3}$. Domain 1, 'Discovery', may be driven by a 'technology push', where an existing imaging technology is applied to an unmet clinical need, or by a 'clinical pull', where the OIB and/or technology are developed in response to a clearly defined unmet clinical need ${ }^{159}$.

In all domains, optical-device development plays a larger role in translation than in standard radiological imaging, where a device to measure the imaging biomarker is usually already clinically approved. The OIB roadmap further emphasizes the concurrency of technical and biological validation. Technical validation seeks to define the precision and accuracy with which a given OIB can be measured, whereas biological and clinical validation seek to connect the OIB with underlying pathological processes and define clinical-performance characteristics such as negative predictive value or specificity. Throughout the OIB roadmap, there is a complex interplay of limitations imposed by the device, the contrast mechanism and the OIB definition, meaning that technical and biological validation cannot be considered in isolation. For example, the precision (repeatability and reproducibility) of measuring a given OIB defined with respect to a perfect test target will differ considerably from the precision defined with respect to a biological measurement made in a patient, which is directly relevant in the clinical application.

The OIB roadmap emphasizes the feedback loops that often inhibit translation, and highlights the key translational characteristics that can be exploited to accelerate translation. Also, our case studies revealed five key translational barriers that need to be addressed to facilitate a smoother path to clinical translation. We discuss each of them in the following subsections.

Lack of defined safe exposure limits for diagnostics. Safe and effective illumination levels that allow accurate measurement of a given biomarker must be established before in vivo trials take place. Visible and near-infrared light is not ionizing, but can lead to thermal damage, photosensitization and photoallergic reactions under certain conditions ${ }^{160}$. Molecularly damaging radiation extends further towards the visible spectrum ${ }^{161}$. The published guidelines for opticalradiation exposure limits only concern the retina and skin and are limited in their application to workers rather than patients, which presents a challenge for those using incoherent light and internal diagnostic-imaging devices ${ }^{160,162}$. Rather, safety for internal exposure is defined in terms of thermal and photobiological consequences, with the assumption that the gastrointestinal tract and skin respond similarly. There is an unmet need for experimentally validated exposure limits for tissues beyond the retina and the skin, so that safe patient exposures for optical imaging can be clearly defined. This would also address frequently raised concerns by ethical review boards of clinical trials. Access to advisors of optical-radiation protection with training in clinical optical imaging would increase the understanding of the capabilities, strengths and weaknesses of optical-imaging systems and their biomarkers. 
Lack of standardized quality assurance. The uncertainty regarding optical-exposure limits is illustrative of a more general lack of standardization in optical imaging. Defining the performance of a device at the point of manufacture and throughout lifetime is crucial for comparing different devices (by measuring the same source of optical contrast) and for debugging device-specific problems.

Whilst performance standards (documents that suggest relevant performance characteristics and test methods) exist for the established non-optical imaging methods, such as the NEMA (National Electrical Manufacturers Association) imaging standards for positron emission tomography (PET) $)^{163}$, optical imaging currently lacks published standards ${ }^{140}$. As a result, optical-imaging contrast agents and devices must often be approved in tandem. This doesn't occur with PET, for which contrast agents are usually approved for use in a range of devices (and PET devices can also be approved for use with a range of contrast agents), because sufficient evidence supporting similarities across devices exists ${ }^{140}$. Unfortunately, the consequence for optical imaging is that healthcare providers must purchase specific devices when working with specific optical-imaging agents, resulting in prohibitive costs and hindering the translation of new contrast agents.

In the United States and Europe, medical devices may be approved based on similarity to a predicate device, gaining 510(k) clearance in the United States and CE marking in the European Union (ref. ${ }^{135}$ ). Yet without standardization, the similarity to a predicate device may be difficult to define. With imaging standards, device performance could be compared to an appropriate standard, allowing inferiority to be spotted at an earlier stage in the translational roadmap. Additionally, if device-performance standards were established it would help separate device approval from agent approval in cases where exogenous agents are being imaged.

Calibration standards exist for all standard diagnostic imaging tests ${ }^{164}$. Stable luminescent phantoms, calibrated to the International System of Units unit of radiance, with an effective shelf life of more than two years, have now been developed to assess some optical diagnostic-imaging tests based on reflectance and fluorescence imaging ${ }^{165}$. Future work in other modalities, such as OCT and photoacoustic imaging, should aim to create similar standards for assessing intra-device and inter-device variabilities, thus helping to achieve improved repeatability and reproducibility for multicentre use.

Lack of validated representative ex vivo models. When tissue is excised from the body, several OIBs change irreversibly. For example, the lack of active blood flow changes the spectrum of tissue by reducing blood oxygenation to $0 \%$, which consequently alters the haemoglobin absorption spectrum. Furthermore, tissue autofluorescence can be modified upon exposure to ambient light, and the tissue structure may be distorted by surgical trauma or by positioning the tissue on a rigid surface. Ultimately, tissues will degrade unless they are frozen or fixed, which further alters tissue properties $^{60}$. The gap between data acquired with ex vivo imaging and data acquired with in vivo imaging is therefore large, and data acquired from ex vivo tissues may contain insurmountable artefacts if the tissues are not properly handled.

Challenges with using ex vivo tissue for validation raise the need for new model systems, which may arise through improved tissue-mimicking phantoms, or from bioengineered tissues. Whichever route is chosen, to avoid disappointing in vivo results later in the roadmap, it is crucial to ensure that the ex vivo model is as representative as possible of the in vivo situation. For example, modalities that are sensitive to blood oxygenation should never use excised tissue for validation unless it is perfused, modalities sensitive to preparation artefacts should avoid chemically fixed or frozen tissue $^{60}$, and dyes that incorporate labelled human antibodies should use models that try to replicate human biology, such as human-derived tumour xenografts, rather than animal tumour models where the binding affinity may be different.

Lack of accurate and validated clinical gold-standards. The clinical gold-standards used as comparators for evaluating the diagnostic accuracy, sensitivity and specificity of new OIBs must be well validated. Most commonly, goldstandard diagnosis is determined via the assessment of stained tissue sections by a pathologist. To achieve the most accurate gold standard for validation of OIBs, consensus of several independent pathologists is needed, as they are not always in agreement ${ }^{166}$. In the best-case scenario, only unanimous decisions would be accepted, although this is difficult to achieve in practice as it significantly decreases the number of samples that can be incorporated into any performance analysis. In practice, decision algorithms are used to combine contrasting histologic diagnoses of several pathologists to reach a single final diagnosis ${ }^{24}$. In future, machine-learning algorithms trained on huge datasets may be able to provide an objective diagnosis, but this is still some way from being realized. Another confounding factor is the transition from a biopsy read to in vivo imaging, which requires an appreciation of nuances associated with the fact that a sample of tissue is typically a mix of pathological and healthy tissue. Each optical imaging pixel could include contributions from one or more tissue states. Validating imaging tools in vivo thus requires high congruence in spatial alignment between the in vivo and ex vivo coordinates, which is not always achieved in basic 'single read' pathology studies. Once the gold standard is validated, careful thought must be applied to how these findings will be accurately co-registered with optical images, so as not to introduce further artefacts into the comparison between the novel technique and the gold standard. The different scales at which in vivo optical-image data is recorded and histopathological analysis is performed can make this particularly challenging ${ }^{167}$.

Difficulties in conducting representative single-centre trials. To maximize opportunities for validation in multicentre trials, early-stage single-centre trials should replicate the common clinical environment as much as possible. First, representative populations should be chosen to reduce spectrum effects ${ }^{168}$. For example, due to the ethical considerations of taking biopsies from healthy tissue, many skin-imaging trials have been carried out in enriched populations with high disease incidence, which has prompted regulatory bodies to disregard such results and investigators to endorse the need for a mix of lesions representative of the target population, for the future testing of new approaches ${ }^{24}$. Second, standard operating procedures should be determined by a single centre and adhered to in multicentre trials in order to prevent bias. For example, inspecting images for minutes when the eventual standard operating procedure in a clinical setting would require inspection of videos in real time results in misleading performance evaluations ${ }^{147-150}$. If image data were collected according to an appropriate standard operating procedure, collation of 
data into an online repository would assist in the validation process for a new OIB. Third, the expertise of the operator and of the image interpreter should be representative of the expertise realistically available in routine care. Endoscopic trimodal imaging provides a cautionary example: promising results in a tertiary referral centre ${ }^{113}$ were not reproduced in a community practice setting ${ }^{111}$, which could have been due to a combination of spectrum effects, different standard operating procedures, or different expertise.

\section{Outlook}

Many of the OIBs reviewed here are still in development; and yet, several are already impacting patient care. Owing to their relatively low-cost implementation, lack of ionizing radiation, and potential for real-time analysis across a range of length scales, OIBs bring new opportunities for changing healthcare practice in the two case studies discussed in this Perspective as well as in many other areas ${ }^{169,170 .}$

Traditionally, the most advanced optical-imaging methods and almost all radiological-imaging methods have been confined to an expert setting, because of the expense and size of these devices and of the need of on-site expertise. The emergence of inexpensive and compact optical-detector technology and communications networks capable of broadcasting images means that image acquisition and interpretation no longer need to be co-localized. The development of cheap, robust and simple-to-use devices makes OIB data acquisition potentially feasible in primary care, for example with tethered capsule endoscopes or handheld skin-imaging devices ${ }^{17,129}$. In addition, the feasibility of image acquisition being performed in one centre, with experts interpreting images remotely, has already been demonstrated in endomicroscopy ${ }^{171}$. OIBs could, in future, be implemented in the home. The rapidly improving specifications of sensors within smartphones, the growing computational power of such devices, and their widespread access to mobile communication networks, have already led to applications for the monitoring of suspicious moles, but so far without proven accuracy or application value ${ }^{172}$. Data captured from smartphones could ultimately be interpreted remotely via expert-image interpreters or by using automated algorithms trained on the vast database of images resulting from such widespread acquisition. Examples of how these changes might impact the case studies discussed here are shown in Fig. 3 .

We expect that this kind of flexible applications of OIBs will rapidly become more common in research. Remote image acquisition and interpretation in a range of indications and healthcare settings, from telepathology ${ }^{173}$ to teledermatology ${ }^{174}$, and from the home to specialist care, are likely to become increasingly widespread, enabling better patient triage and decreasing the costs associated with high-volume specialist-care imaging suites and with expert operators. Remote interpretation will allow for greater division of expertise, reducing training costs, and increasing the average expertise available for image analysis. For some indications, automated diagnosis trained on centralized image databases might circumvent the need for an image interpreter entirely.

There are a number of challenges for advancing the clinical implementation of OIBs. Regulatory approvals for new optical-imaging devices tend to favour commercial vendors rather than clinical value ${ }^{135}$, and often procedures are approved with a new device where the application is not improving care but rather just changing the sub-specialty that implements the procedure. Commercially, devices may be approved on the basis of similarity to a predicate device, without proof of clinical utility ${ }^{135}$. Without optical-imaging standardization, similarity to a predicate may be difficult to define. Worse still is the prospect that the predicate itself may pre-date current regulations. Since devices can be marketed without proof of clinical utility, manufacturers may lack the motivation to invest the significant time, money and expertise required to conduct trials for assessing clinical utility, despite the potential long-term benefits.

Distributing devices into the hands of the patients themselves raises questions about the appropriate nature of clinical trials for testing, and brings additional challenges in standardization. Although the individual optical hardware or software may itself be inexpensive, the final clinical test must fully represent the cost of development, meaning that the cost-pertest can in some cases be similar to or greater than the costs of the existing standard of care.

The advent of new liquid biomarkers has raised questions about the future utility of imaging in the context of early cancer detection. Imaging currently provides crucial insight for the early detection of malignant melanoma and oesophageal cancer, and endoscopy methods are also broadly applied for the identification of cancers in the aerodigestive tract, as well as in the cervix and bladder. Although liquid biomarkers have shown great promise, even a study ${ }^{175}$ that achieved good performance for the detection of late-stage cancers ( $73 \%$ sensitivity) reported much poorer performance for the detection of early-stage disease ( $40 \%$ median sensitivity in the eight cancers tested, and only $20 \%$ sensitivity in oesophageal cancer). Moreover, although liquid biomarkers have the potential to accurately detect and even localize early-stage disease, imaging is likely to remain an important tool in screening and surveillance programmes for early cancer detection for many years to come. Also, imaging is the only test capable of providing the detailed spatially resolved information that is ultimately required for the resection of early cancers, and has shown great promise in this regard $^{176,177}$. It is therefore likely that liquid biomarkers could be implemented as tools complementary to imaging, perhaps as a low-cost early-stage option to pre-screen patients prior to imaging, should a sufficiently high specificity be achieved.

Advanced optical-imaging research requires the technical development of optical devices as well as the technical and biological validation of the resulting OIBs. In many cases, the discovery of promising OIBs using optical devices dedicated to ex vivo imaging can motivate the development of devices able to measure the same biomarker in vivo. An understanding of the strengths and weaknesses of these optical devices is needed to assess the feasibility of measuring certain biomarkers in a clinical scenario, with a clear appreciation for the biological heterogeneity of the biomarker. For example, it may be the case that a multimodal approach is needed to achieve the required performance through the combination of two or more OIBs (ref. ${ }^{178}$ ). These aspects demand a multidisciplinary research effort that combines 
clinical expertise of an unmet need with physics and engineering knowledge of the optical interactions in order to specifically tailor devices for the detection of known biomarkers. The sharing of intellectual-property ownership, or of appropriate licencing agreements, may also be required in order to combine imaging modalities. Furthermore, collaboration with computer scientists may help to better the design of interpretation algorithms to yield useful clinical biomarkers. Embracing this multidisciplinary approach is necessary to promote the translation of OIBs.

Imaging is currently the only medical tool capable of providing detailed, immediate and spatially resolved biological information in vivo, which is especially relevant for the diagnosis of the earliest stages of cancer. Despite these promises, few OIBs have been translated to routine clinical use. The translational characteristics and barriers discussed here complement the OIB roadmap, which we hope will improve the chances of new optical-imaging approaches achieving widespread clinical implementation. The vast array of complex tissue-light interactions and the equally diverse arsenal of optical devices for detecting these interactions should help to improve the current standard-of-care for cancer patients.

\section{Acknowledgements}

We would like to thank Fiona Walter of the University of Cambridge for helpful comments on our manuscript. D.J.W., C.R.M.F. and S.E..B. are financially supported by CRUK (C14303/A17197, C47594/A16267, C47594/A21102, C55962/A24669) and EPSRC (C197/A16465, EP/N014588/1).

\section{Author contributions}

D.J.W, J.P.B.O. and S.E.B. conceived the manuscript. D.J.W. researched and wrote the manuscript together with C.R.M.F. B.W.P. reviewed and edited the manuscript. All authors discussed and agreed with the final version of the manuscript.

\section{Competing interests}

S.E.B. receives research support from iThera Medical $\mathrm{GmbH}$ and PreXion Inc., and chairs the International Photoacoustic Standardisation Consortium (IPASC). 
Fig. 1 | Visible and near-infrared light-tissue interactions. Visible and near-infrared light experiences a wide range of complex interactions with tissue constituents. OIBs exploit these interactions in clinical applications. The interactions illustrated are: reflection, absorption and scattering (occurring, for example, in white-light imaging, diffuse reflectance spectroscopy, elastic scattering spectroscopy, narrow-band imaging, and multispectral or hyperspectral imaging), phase (in, for example, OCT and angle-resolved low-coherence interferometry), polarization (in, for example, polarimetry and holography), nonlinear effects (in Raman spectroscopy, coherent anti-stokes Raman spectroscopy, multiphoton fluorescence imaging and multi-harmonic generation imaging), fluorescence (in autofluorescence intensity or lifetime imaging of endogenous fluorophores, and in optical molecular imaging of exogenous fluorophores), and the photoacoustic effect (in photoacoustic, or optoacoustic, microscopy and tomography). Line colours represent wavelengths of light. Multicoloured lines represent broadband light. Changes in colour represent changes in wavelength when light interacts with tissue. Perpendicular lines represent wavefronts and thus indicate the optical coherence of phase. Arrowed perpendicular lines represent the orientation of polarization. Curved lines represent the emission of acoustic waves. ICG, indocyanine green; IV, intravenous; $\mathrm{NAD}(\mathrm{P}) \mathrm{H}=$ nicotinamide adenine dinucleotide (phosphate).

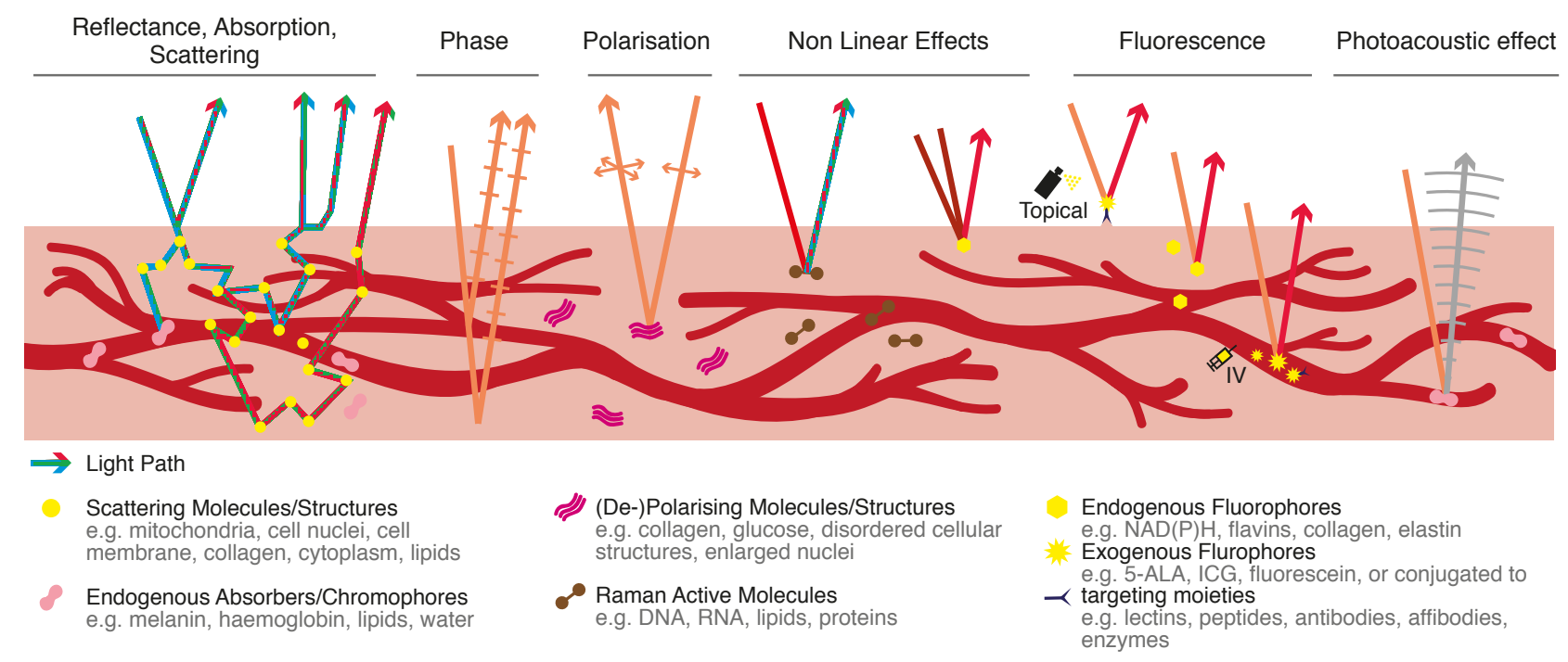


Fig. 2 | Roadmap for OIBs. The proposed roadmap differs from the radiological-imaging-biomarker roadmap ${ }^{3}$ by considering the intrinsic coupling of OIBs to the development of the optical-imaging modality. Red feedback loops decelerate clinical translation. Purple circles represent points at which careful consideration of translational characteristics (1-6) can accelerate translation. Technical and biological validations occur in parallel, and are closely linked because of the interplay between device, operator, interpreter and OIB. The technical validation occurs throughout the roadmap to ensure availability and precision in all settings. Cost effectiveness is omitted here yet it impacts the roadmap at every stage, owing to the equipment and personnel costs of performing imaging studies ${ }^{3}$. Translational characteristics: (1) contrast mechanism, (2) instrumentation, (3) operator expertise, (4) image-interpreter expertise, (5) repeatability and reproducibility, and (6) co-registration. SOP, standard operating procedure.

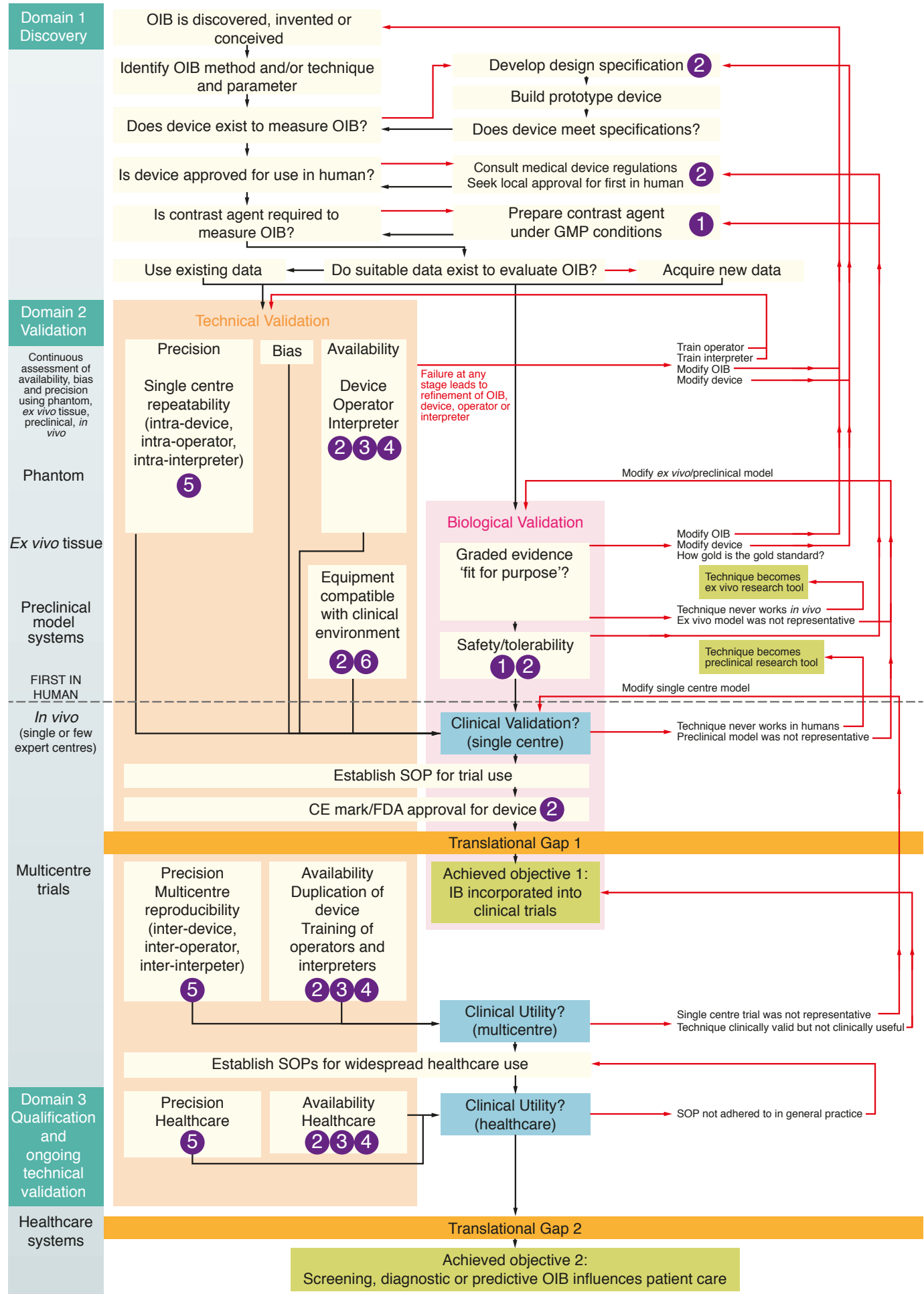


Fig. 3 | Current and potential future clinical implementations of optical imaging in two case studies. The current clinical pipeline (black arrows) from the home through primary care to specialist care is shown for patients with suspected Barrett's oesophagus (green) and suspicious malignant melanoma (pink). The potential future pipelines (red lines) will be mediated by new or modified indications for imaging (red boxes) and high-speed communication networks (red dashed lines). Risk stratification in primary care reduces the number of false positives referred for urgent specialist follow-up. Boxes highlight where imaging takes place in the pipeline.

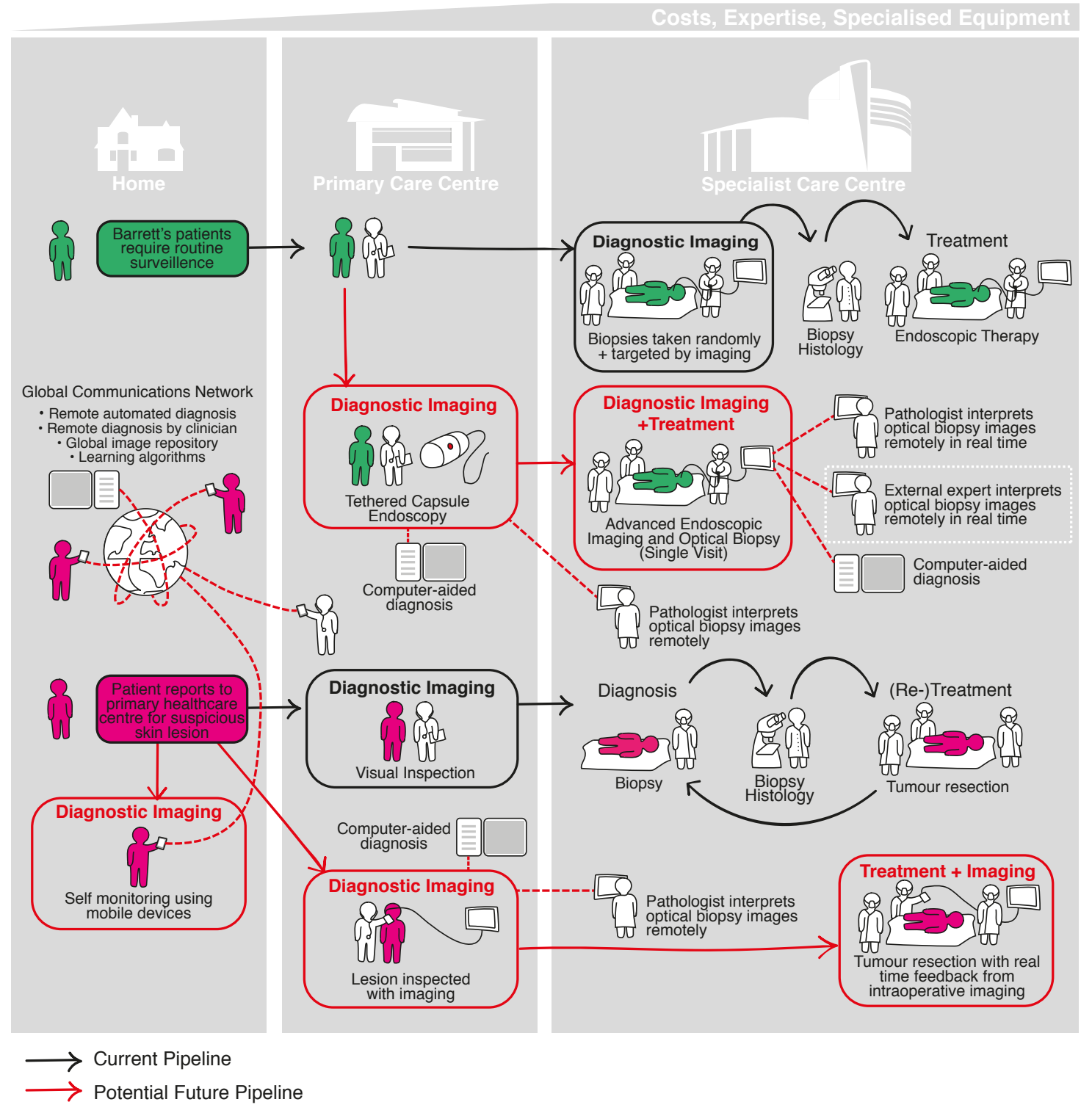


Table 1 | Optical-imaging modalities, and selected OIBs currently in development for early cancer detection.

\begin{tabular}{|c|c|c|c|c|c|}
\hline Approach & Source of contrast & $\begin{array}{l}\text { Biological } \\
\text { change in cancer }\end{array}$ & Example of OIB & $\begin{array}{l}\text { Clinically approved } \\
\text { commercial devices }\end{array}$ & Refs. \\
\hline \multicolumn{6}{|c|}{ Wide-field reflectance imaging } \\
\hline $\begin{array}{l}\text { White-light imaging } \\
\text { (standard of care) }\end{array}$ & \multirow{2}{*}{$\begin{array}{l}\text { Endogenous } \\
\text { chromophores (such as } \\
\text { melanin, haemoglobin, } \\
\text { tryptophan, lipids, water); } \\
\text { endogenous scatterers } \\
\text { (such as mitochondria, } \\
\text { cell nuclei, and cell } \\
\text { membrane) }\end{array}$} & \multirow[b]{2}{*}{$\begin{array}{l}\text { Vasculature, } \\
\text { melanin } \\
\text { distribution, visible } \\
\text { lesions }\end{array}$} & $\begin{array}{l}\text { Mucosal } \\
\text { abnormalities }\end{array}$ & $\begin{array}{l}\text { Widely available } \\
\text { endoscopes and } \\
\text { dermoscopes, naked eye } \\
\text { examination }\end{array}$ & 179 \\
\hline $\begin{array}{l}\text { Virtual (or } \\
\text { electronic) } \\
\text { chromoendoscopy }\end{array}$ & & & $\begin{array}{l}\text { Irregular mucosal } \\
\text { and vascular } \\
\text { patterns }\end{array}$ & $\begin{array}{l}\text { Olympus NBI Scopes (ref. } \\
{ }^{180} \text { ), Fujicon FICE (ref. }{ }^{181} \text { ). } \\
\text { PENTAX iScan (ref. }{ }^{182} \text { ), } \\
\text { SIMSYS-MoleMate (ref. } \\
{ }^{183} \text { ). MelaFind (ref. }{ }^{184} \text { ), } \\
\text { Spectral Molecular } \\
\text { Imaging SkinSpect (ref. } \\
{ }^{185} \text { ) }\end{array}$ & $\begin{array}{l}5,156,186- \\
188\end{array}$ \\
\hline Polarimetry & Microstructural anisotropy & $\begin{array}{l}\text { Collagen, cellular } \\
\text { orientations, } \\
\text { organization, } \\
\text { muscular } \\
\text { hypertrophy and } \\
\text { muscular atrophy }\end{array}$ & Unclear & $\begin{array}{l}\text { Derma Medical MoleMax } \\
3 \text { (ref. }{ }^{189} \text { ) }\end{array}$ & 190 \\
\hline
\end{tabular}

Dye-based imaging

\begin{tabular}{|c|c|c|c|c|c|}
\hline Chromoendoscopy & $\begin{array}{l}\text { Dyes or stains (such as } \\
\text { methylene blue and acetic } \\
\text { acid). Can enhance } \\
\text { surface topology or be } \\
\text { differentially absorbed by } \\
\text { different cell types }\end{array}$ & $\begin{array}{l}\text { Visible lesions, } \\
\text { cell type }\end{array}$ & $\begin{array}{l}\text { Loss of aceto- } \\
\text { whitening }\end{array}$ & $\begin{array}{l}\text { Widely available } \\
\text { endoscopes }\end{array}$ & 5,188 \\
\hline $\begin{array}{l}\text { Targeted } \\
\text { fluorescence } \\
\text { imaging }\end{array}$ & $\begin{array}{l}\text { Exogenous fluorophores } \\
\text { conjugated to targeting } \\
\text { moieties (lectins, } \\
\text { peptides, antibodies, } \\
\text { affibodies and enzymes) } \\
\text { that target intracellular } \\
\text { and extracellular proteins } \\
\text { and enzymes }\end{array}$ & $\begin{array}{l}\text { Changes in } \\
\text { targeted } \\
\text { intracellular and } \\
\text { extracellular } \\
\text { proteins and } \\
\text { enzymes }\end{array}$ & $\begin{array}{l}\text { High-intensity } \\
\text { fluorescence, due to } \\
\text { uptake or binding of } \\
\text { probe }\end{array}$ & None & 76,86 \\
\hline $\begin{array}{l}\text { Endoscopic } \\
\text { microscopy }\end{array}$ & $\begin{array}{l}\text { Exogenous fluorophores, } \\
\text { such as fluorescein }\end{array}$ & Cell type & $\begin{array}{l}\text { Change in cell } \\
\text { phenotype, such as } \\
\text { irregular borders and } \\
\text { shapes }\end{array}$ & $\begin{array}{l}\text { Mauna Kea Cellvizio (ref. } \\
{ }^{191} \text { ), Optiscan ViewnVivo } \\
\text { (ref. }{ }^{192} \text { ), Caliber ID } \\
\text { VivaScope (ref. }{ }^{193} \text { ) }\end{array}$ & 5,40 \\
\hline
\end{tabular}

Autofluorescence imaging

\begin{tabular}{|c|c|c|c|c|c|}
\hline $\begin{array}{l}\text { Autofluorescence } \\
\text { intensity imaging }\end{array}$ & $\begin{array}{l}\text { Endogenous fluorophores } \\
\text { (such as NAD(P)H, }\end{array}$ & Biochemical, such & $\begin{array}{l}\text { Loss of } \\
\text { autofluorescence, } \\
\text { denoted by purple } \\
\text { image highlight }\end{array}$ & $\begin{array}{l}\text { Olympus GIF-FQ20Z194, } \\
\text { CF-FH260AZL/I }{ }^{195}, \mathrm{BF}^{-} \\
\text {F260 }\end{array}$ & 197 \\
\hline $\begin{array}{l}\text { Fluorescence } \\
\text { lifetime imaging }\end{array}$ & $\begin{array}{l}\text { flavins, collagen, elastin, } \\
\text { phenylalanine and } \\
\text { tryptophan) }\end{array}$ & $\begin{array}{l}\text { as collagen- } \\
\text { crosslink breaking }\end{array}$ & $\begin{array}{l}\text { Change in } \\
\text { melanocyte } \\
\text { distribution }\end{array}$ & $\begin{array}{l}\text { JenLab Dermalnspect } \\
\text { (ref. }{ }^{198} \text { ) }\end{array}$ & 199-201 \\
\hline $\begin{array}{l}\text { Multi Photon } \\
\text { Microscopy }\end{array}$ & & & $\begin{array}{l}\text { Melanocyte } \\
\text { distribution }\end{array}$ & $\begin{array}{l}\text { JenLab Dermalnspect } \\
\text { (ref. }{ }^{198} \text { ) }\end{array}$ & 199-201 \\
\hline
\end{tabular}

Cross-sectional imaging

\begin{tabular}{|c|c|c|c|c|c|}
\hline $\begin{array}{l}\text { Optical coherence } \\
\text { tomography }\end{array}$ & $\begin{array}{l}\text { Boundaries between } \\
\text { structural features }\end{array}$ & $\begin{array}{l}\text { Vasculature, } \\
\text { visible lesions, } \\
\text { structural changes }\end{array}$ & $\begin{array}{l}\text { Change in structure, } \\
\text { such as irregular } \\
\text { glandular } \\
\text { architecture }\end{array}$ & $\begin{array}{l}\text { Ninepoint Medical } \\
\text { NvisionVLE (ref. }{ }^{202} \text { ), } \\
\text { Verisante Core (ref. }{ }^{203} \text { ), } \\
\text { Skintell (ref. }{ }^{41} \text { ), Michelson } \\
\text { Diagnostics Vivosight (ref. }\end{array}$ & 205 \\
\hline
\end{tabular}


204)

\begin{tabular}{llll}
$\begin{array}{lll}\text { Photoacoustic } \\
\text { imaging }\end{array}$ & $\begin{array}{l}\text { Absorption of endogenous } \\
\text { chromophores (such as } \\
\text { melanin and } \\
\text { haemoglobin) }\end{array}$ & $\begin{array}{l}\text { Reduction in oxygen } \\
\text { saturation }\end{array}$ & None \\
\hline
\end{tabular}

Spectroscopy

\begin{tabular}{lllll}
\hline $\begin{array}{l}\text { Raman } \\
\text { spectroscopy }\end{array}$ & $\begin{array}{l}\text { Vibrational modes of } \\
\text { specific molecules (such } \\
\text { as proteins, DNA and } \\
\text { lipids) }\end{array}$ & Biochemical & Change in spectrum & Verisante Aura (ref. ${ }^{206}$ ) \\
\hline $\begin{array}{l}\text { Reflection } \\
\text { spectroscopy }\end{array}$ & $\begin{array}{l}\text { Reflection spectra of } \\
\text { endogenous biological } \\
\text { molecules }\end{array}$ & Biochemical & Change in spectrum & $\begin{array}{l}\text { Pentax WavSTAT4 (ref. 210) } \\
\text { * }\end{array}$ \\
\hline $\begin{array}{l}\text { Diffuse reflectance } \\
\text { spectroscopy }\end{array}$ & $\begin{array}{l}\text { Endogenous scatterers } \\
\text { (such as mitochondria, } \\
\text { cell nuclei, cell } \\
\text { membrane, collagen, } \\
\text { cytoplasm and lipids) }\end{array}$ & $\begin{array}{l}\text { Vasculature, } \\
\text { change in } \\
\text { distribution of } \\
\text { cells, nuclei and } \\
\text { organelles }\end{array}$ & Change in spectrum & None \\
\hline
\end{tabular}

FOV, field of view. *Lower gastrointestinal cancers. 


\section{References}

1. A. Wax et al., "Angle-resolved low coherence interferometry for detection of dysplasia in Barrett's esophagus.," Gastroenterology 141(2), 443-447 (2011) [doi:10.1053/j.gastro.2011.06.020].

2. Y. Imamoto and Y. Shichida, "Cone visual pigments," Biochim. Biophys. Acta - Bioenerg. 1837(5), 664-673, Elsevier B.V. (2014) [doi:10.1016/j.bbabio.2013.08.009].

3. J. P. B. O'Connor et al., "Imaging biomarker roadmap for cancer studies," Nat. Rev. Clin. Oncol. 14(3), 169-186 (2017) [doi:10.1038/nrclinonc.2016.162].

4. P. Sharma et al., "White Paper AGA: Advanced Imaging in Barrett's Esophagus," Clin. Gastroenterol. Hepatol. 13(13), 2209-2218, Elsevier, Inc (2015) [doi:10.1016/j.cgh.2015.09.017].

5. N. Thosani et al., "ASGE Technology Committee systematic review and meta-analysis assessing the ASGE Preservation and Incorporation of Valuable Endoscopic Innovations thresholds for adopting real-time imagingassisted endoscopic targeted biopsy during endoscopic surveillance," Gastrointest. Endosc. 83(4), 684-698, Elsevier, Inc. (2016) [doi:10.1016/j.gie.2016.01.007].

6. V. Nikolaou and A. J. Stratigos, "Emerging trends in the epidemiology of melanoma," Br. J. Dermatol. 170(1), 11-19 (2014) [doi:10.1111/bjd.12492].

7. NICE Guideline, "Suspected Cancer: Recognition and Referral," NICE Guidel.(June), 1-378 (2015).

8. V. Terushkin and A. C. Halpern, "Melanoma Early Detection," Hematol. Oncol. Clin. North Am. 23(3), 481-500 (2009) [doi:10.1016/j.hoc.2009.03.001].

9. C. Lindholm et al., "Invasive cutaneous malignant melanoma in Sweden, 1990-1999: A prospective, populationbased study of survival and prognostic factors," Cancer 101(9), 2067-2078 (2004) [doi:10.1002/cncr.20602].

10. E. W. Breitbart et al., "Systematic skin cancer screening in Northern Germany," J. Am. Acad. Dermatol. 66(2), 201-211, Elsevier Inc (2012) [doi:10.1016/j.jaad.2010.11.016].

11. A. Katalinic et al., "Does skin cancer screening save lives?: An observational study comparing trends in melanoma mortality in regions with and without screening," Cancer 118(21), 5395-5402 (2012) [doi:10.1002/cncr.27566].

12. K. Bibbins-Domingo et al., "Screening for Skin Cancer," Jama 316(4), 429-435 (2016) [doi:10.1001/jama.2016.8465].

13. A. J. Stratigos et al., "Euromelanoma: A dermatology-led European campaign against nonmelanoma skin cancer and cutaneous melanoma. Past, present and future," Br. J. Dermatol. 167(SUPPL. 2), 99-104 (2012) [doi:10.1111/j.1365-2133.2012.11092.x].

14. M. E. Vestergaard et al., "Dermoscopy compared with naked eye examination for the diagnosis of primary melanoma: A meta-analysis of studies performed in a clinical setting," Br. J. Dermatol. 159(3), 669-676 (2008) [doi:10.1111/j.1365-2133.2008.08713.x].

15. M. Moncrieff et al., "Spectrophotometric intracutaneous analysis: a new technique for imaging pigmented skin lesions," Br. J. Dermatol. 146(3), 448-457 (2002) [doi:10.1046/j.1365-2133.2002.04569.x].

16. J. D. Emery et al., "Accuracy of SIAscopy for pigmented skin lesions encountered in primary care : development and validation of a new diagnostic algorithm" (2010).

17. F. M. Walter et al., "Protocol for the MoleMate UK Trial: a randomised controlled trial of the MoleMate system in the management of pigmented skin lesions in primary care [ISRCTN 79932379].," BMC Fam. Pract. 11, 36 (2010) [doi:10.1186/1471-2296-11-36].

18. F. M. Walter et al., "Effect of adding a diagnostic aid to best practice to manage suspicious pigmented lesions in primary care: randomised controlled trial.," Br. Med. J. 345(July), e4110 (2012) [doi:10.1136/bmj.e4110].

19. F. Vasefi et al., "Multimode optical dermoscopy (SkinSpect) analysis for skin with melanocytic nevus," in Proc. of SPIE 9711, p. 971110 (2016) [doi:10.1117/12.2214288].

20. F. Vasefi et al., "Polarization-Sensitive Hyperspectral Imaging in vivo: A Multimode Dermoscope for Skin Analysis," Sci. Rep. 4, 4924 (2014) [doi:10.1038/srep04924].

21. F. Vasefi et al., "Separating melanin from hemodynamics in nevi using multimode hyperspectral dermoscopy and spatial frequency domain spectroscopy," J. Biomed. Opt. 21(11), 114001 (2016) [doi:10.1117/1.JBO.21.11.114001].

22. N. MacKinnon et al., "In vivo skin chromophore mapping using a multimode imaging dermoscope (SkinSpect)," Imaging, Manip. Anal. Biomol. Cells, Tissues XI 8587, 1-13 (2013) [doi:85870u 10.1117/12.2005587].

23. M. Elbaum et al., "Automatic differentiation of melanoma from melanocytic nevi with multispectral digital dermoscopy: A feasibility study," J. Am. Acad. Dermatol. 44(2), 207-218 (2001) [doi:10.1067/mjd.2001.110395].

24. G. Monheit et al., "The performance of MelaFind: a prospective multicenter study.," Arch. Dermatol. 147(2), 188- 
194 (2011) [doi:10.1001/archdermatol.2010.302].

25. A. Hauschild et al., "To excise or not: impact of MelaFind on German dermatologists' decisions to biopsy atypical lesions," JDDG J. der Dtsch. Dermatologischen Gesellschaft 12(7), 606-614 (2014) [doi:10.1111/ddg.12362].

26. Y.-Q. Xiong et al., "Comparison of dermoscopy and reflectance confocal microscopy for the diagnosis of malignant skin tumours: a meta-analysis," J. Cancer Res. Clin. Oncol., Springer Berlin Heidelberg (2017) [doi:10.1007/s00432-017-2391-9].

27. S. Segura et al., "Development of a two-step method for the diagnosis of melanoma by reflectance confocal microscopy," J. Am. Acad. Dermatol. 61(2), 216-229, Elsevier Inc (2009) [doi:10.1016/j.jaad.2009.02.014].

28. P. Guitera et al., "Surveillance for treatment failure of lentigo maligna with dermoscopy and in vivo confocal microscopy: New descriptors," Br. J. Dermatol. 170(6), 1305-1312 (2014) [doi:10.1111/bjd.12839].

29. P. Guitera et al., "In vivo reflectance confocal microscopy enhances secondary evaluation of melanocytic lesions.," J. Invest. Dermatol. 129(1), 131-138, Elsevier Masson SAS (2009) [doi:10.1038/jid.2008.193].

30. I. Stanganelli et al., "Integration of reflectance confocal microscopy in sequential dermoscopy follow-up improves melanoma detection accuracy," Br. J. Dermatol. 172(2), 365-371 (2015) [doi:10.1111/bjd.13373].

31. A. M. Witkowski et al., "Non-invasive diagnosis of pink basal cell carcinoma: How much can we rely on dermoscopy and reflectance confocal microscopy?," Ski. Res. Technol. 22(2), 230-237 (2016) [doi:10.1111/srt.12254].

32. M. Venturini et al., "Reflectance confocal microscopy allows in vivo real-time noninvasive assessment of the outcome of methyl aminolaevulinate photodynamic therapy of basal cell carcinoma," Br. J. Dermatol. 168(1), 99105 (2013) [doi:10.1111/bjd.12052].

33. R. G. B. Langley et al., "The diagnostic accuracy of in vivo confocal scanning laser microscopy compared to dermoscopy of benign and malignant melanocytic lesions: a prospective study.," Dermatology 215, 365-372 (2007) [doi:10.1159/000109087]

34. E. Moscarella et al., "The role of reflectance confocal microscopy as an aid in the diagnosis of collision tumors," Dermatology 227(2), 109-117 (2013) [doi:10.1159/000351771]

35. I. Alarcon et al., "Impact of in vivo reflectance confocal microscopy on the number needed to treat melanoma in doubtful lesions," Br. J. Dermatol. 170(4), 802-808 (2014) [doi:10.1111/bjd.12678].

36. NICE, "VivaScope 1500 and 3000 imaging systems for detecting skin cancer lesions - guidance (DG19)" (2015).

37. K. Konig and I. Riemann, "High-resolution multiphoton tomography of human skin with subcellular spatial resolution and picosecond time resolution.," J. Biomed. Opt. 8(3), 432-439 (2003) [doi:10.1117/1.1577349].

38. M. Manfredini et al., "High-resolution imaging of basal cell carcinoma: A comparison between multiphoton microscopy with fluorescence lifetime imaging and reflectance confocal microscopy," Ski. Res. Technol. 19(1), 433-443 (2013) [doi:10.1111/j.1600-0846.2012.00661.x].

39. S. Seidenari et al., "Multiphoton Laser Tomography and Fluorescence Lifetime Imaging of Melanoma: Morphologic Features and Quantitative Data for Sensitive and Specific Non-Invasive Diagnostics," PLoS One 8(7) (2013) [doi:10.1371/journal.pone.0070682].

40. M. Ulrich et al., "Dynamic Optical Coherence Tomography in Dermatology," Dermatology 232(3), 298-311 (2016) [doi:10.1159/000444706]

41. M. A. L. M. Boone et al., "High-definition optical coherence tomography imaging of melanocytic lesions: A pilot study," Arch. Dermatol. Res. 306(1), 11-26 (2014) [doi:10.1007/s00403-013-1387-9].

42. T. Gambichler et al., "High-definition optical coherence tomography of melanocytic skin lesions," J. Biophotonics 8(8), 681-686 (2015) [doi:10.1002/jbio.201400085].

43. S. Schuh et al., "Comparison of different optical coherence tomography devices for diagnosis of non-melanoma skin cancer," Ski. Res. Technol. 22(4), 395-405 (2016) [doi:10.1111/srt.12277].

44. R. C. Nolan et al., "Optical Coherence Tomography for the Neurologist," Semin Neurol, 564-577 (2015) [doi:10.1055/s-0035-1563579].

45. A. C. S. Tan et al., "An overview of the clinical applications of optical coherence tomography angiography," Eye(July), 1-25, Nature Publishing Group (2017) [doi:10.1038/eye.2017.181].

46. Q. Liu, "Role of optical spectroscopy using endogenous contrasts in clinical cancer diagnosis.," World J. Clin. Oncol. 2(1), 50-63 (2011) [doi:10.5306/wjco.v2.i1.50].

47. C. Kendall et al., "Vibrational spectroscopy: a clinical tool for cancer diagnostics," Analyst 134(6), 1029-1045 (2009) [doi:10.1039/b822130h].

48. I. Pence and A. Mahadevan-Jansen, "Clinical instrumentation and applications of Raman spectroscopy," Chem. Soc. Rev. 45(7), 1958-1979, Royal Society of Chemistry (2016) [doi:10.1039/C5CS00581G].

49. L. Brancaleon et al., "In vivo fluorescence spectroscopy of nonmelanoma skin cancer.," Photochem. Photobiol. 
73(2), 178-183 (2001) [doi:10.1562/0031-8655(2001)0730178IVFSON2.0.CO2].

50. A. Garcia-Uribe et al., "Skin cancer detection by spectroscopic oblique-incidence reflectometry: classification and physiological origins.," Appl. Opt. 43(13), 2643-2650 (2004) [doi:10.1364/AO.43.002643].

51. N. Rajaram et al., "Pilot clinical study for quantitative spectral diagnosis of non-melanoma skin cancer," Lasers Surg. Med. 42(10), 716-727 (2010) [doi:10.1002/lsm.21009].

52. A. J. Thompson et al., "In vivo measurements of diffuse reflectance and time-resolved autofluorescence emission spectra of basal cell carcinomas," J. Biophotonics 5(3), 240-254 (2012) [doi:10.1002/jbio.201100126].

53. A. Garcia-Uribe et al., "In vivo diagnosis of melanoma and nonmelanoma skin cancer using oblique incidence diffuse reflectance spectrometry," Cancer Res. 72(11), 2738-2745 (2012) [doi:10.1158/0008-5472.CAN-114027].

54. T. Upile et al., "Elastic scattering spectroscopy in assessing skin lesions: An ' in vivo' study," Photodiagnosis Photodyn. Ther. 9(2), 132-141, Elsevier B.V. (2012) [doi:10.1016/j.pdpdt.2011.12.003].

55. H. Lui et al., "Real-time raman spectroscopy for in vivo skin cancer diagnosis," Cancer Res. 72(10), 2491-2500 (2012) [doi:10.1158/0008-5472.CAN-11-4061].

56. J. Zhao et al., "Real-time Raman spectroscopy for non-invasive skin cancer detection - preliminary results.," Conf. Proc. IEEE Eng. Med. Biol. Soc. 2008, 3107-3109 (2008) [doi:10.1109/IEMBS.2008.4649861].

57. L. Lim et al., "Clinical study of noninvasive in vivo melanoma and nonmelanoma skin cancers using multimodal spectral diagnosis.," J. Biomed. Opt. 19(11), 117003 (2014) [doi:10.1117/1.JBO.19.11.117003].

58. J. Schleusener et al., "In vivo study for the discrimination of cancerous and normal skin using fibre probe-based Raman spectroscopy," Exp. Dermatol. 24(10), 767-772 (2015) [doi:10.1111/exd.12768].

59. V. P. Zakharov et al., "Combined Raman spectroscopy and autofluoresence imaging method for in vivo skin tumor diagnosis," 1-8 (2014) [doi:10.1117/12.2061667].

60. M. G. Shim and B. C. Wilson, "The effects of ex vivo handling procedures on the near-infrared Raman spectra of normal mammalian tissues.," Photochem. Photobiol. 63(5), 662-671 (1996) [doi:10.1111/j.17511097.1996.tb05671.x]

61. S. Kim, K. M. Byun, and S. Y. Lee, "Influence of water content on Raman spectroscopy characterization of skin sample," Biomed. Opt. Express 8(2), 1130-1138 (2017) [doi:10.1364/BOE.8.001130].

62. F. Hvid-Jensen et al., "Incidence of adenocarcinoma among patients with Barrett's esophagus.," N. Engl. J. Med. 365(15), 1375-1383 (2011) [doi:10.1056/NEJMoa1103042].

63. P. Gatenby et al., "Lifetime risk of esophageal adenocarcinoma in patients with Barrett's esophagus.," World J. Gastroenterol. 20(28), 9611-9617 (2014) [doi:10.3748/wjg.v20.i28.9611].

64. L. C. Duits et al., "Barrett's oesophagus patients with low-grade dysplasia can be accurately risk-stratified after histological review by an expert pathology panel.," Gut 64(5), 700-706 (2015) [doi:10.1136/gutjnl-2014-307278].

65. S. Singh et al., "Incidence of esophageal adenocarcinoma in Barrett's esophagus with low-grade dysplasia: a systematic review and meta-analysis.," Gastrointest. Endosc. 79(6), 897-909.e4; quiz 983.e1, 983.e3 (2014) [doi:10.1016/j.gie.2014.01.009].

66. R. C. Fitzgerald et al., "British Society of Gastroenterology guidelines on the diagnosis and management of Barrett's oesophagus.," Gut 63(1), 7-42 (2014) [doi:10.1136/gutjnl-2013-305372].

67. S. J. Spechler et al., "American Gastroenterological Association technical review on the management of Barrett's esophagus.," Gastroenterology 140(3), e18-e52 (2011) [doi:10.1053/j.gastro.2011.01.031].

68. K. K. Wang and R. E. Sampliner, "Updated guidelines 2008 for the diagnosis, surveillance and therapy of Barrett's esophagus.," Am. J. Gastroenterol. 103(3), 788-797 (2008) [doi:10.1111/j.1572-0241.2008.01835.x].

69. J. A. Evans et al., "The role of endoscopy in Barrett's esophagus and other premalignant conditions of the esophagus," Gastrointest. Endosc. 76(6), 1087-1094 (2012) [doi:10.1016/j.gie.2012.08.004].

70. B. Weusten et al., "Endoscopic management of Barrett's esophagus: European Society of Gastrointestinal Endoscopy (ESGE) Position Statement.," Endoscopy (2017) [doi:10.1055/s-0042-122140].

71. J. H. Rubenstein et al., "Effect of a prior endoscopy on outcomes of esophageal adenocarcinoma among United States veterans.," Gastrointest. Endosc. 68(5), 849-855 (2008) [doi:10.1016/j.gie.2008.02.062].

72. G. S. Cooper, T. D. Kou, and A. Chak, "Receipt of previous diagnoses and endoscopy and outcome from esophageal adenocarcinoma: a population-based study with temporal trends.," Am. J. Gastroenterol. 104(6), 1356-1362 (2009) [doi:10.1038/ajg.2009.159].

73. H. B. El-Serag et al., "Surveillance endoscopy is associated with improved outcomes of oesophageal adenocarcinoma detected in patients with Barrett's oesophagus.," Gut 65, 1252-1260 (2016) [doi:10.1136/gutjnl2014-308865].

74. F. Kastelein et al., "Impact of surveillance for Barrett's oesophagus on tumour stage and survival of patients with 
neoplastic progression," Gut 65(4), 1-7 (2015) [doi:10.1136/gutjnl-2014-308802].

75. R. E. Verbeek et al., "Surveillance of Barrett's Esophagus and Mortality from Esophageal Adenocarcinoma: A Population-Based Cohort Study.," Am. J. Gastroenterol. 109(8), 1215-1222, Nature Publishing Group (2014) [doi:10.1038/ajg.2014.156].

76. M. B. Sturm and T. D. Wang, "Emerging optical methods for surveillance of Barrett's oesophagus.," Gut 64, 1816-1823 (2015) [doi:10.1136/gutjnl-2013-306706].

77. F. J. Q. Chedgy et al., "Acetic acid chromoendoscopy: Improving neoplasia detection in Barrett's esophagus," World J. Gastroenterol. 22(25), 5753-5760 (2016) [doi:10.3748/wjg.v22.i25.5753].

78. P. Sharma et al., "The American Society for Gastrointestinal Endoscopy PIVI (Preservation and Incorporation of Valuable Endoscopic Innovations) on imaging in Barrett's Esophagus.," Gastrointest. Endosc. 76(2), 252-254 (2012) [doi:10.1016/j.gie.2012.05.007].

79. C. H. Lim et al., "Randomized crossover study that used methylene blue or random 4-quadrant biopsy for the diagnosis of dysplasia in Barrett's esophagus," Gastrointest. Endosc. 64(2), 195-199 (2006) [doi:10.1016/j.gie.2005.07.025].

80. J. D. Horwhat et al., "A randomized comparison of methylene blue-directed biopsy versus conventional fourquadrant biopsy for the detection of intestinal metaplasia and dysplasia in patients with long-segment Barrett's esophagus.," Am. J. Gastroenterol. 103(3), 546-554 (2008) [doi:10.1111/j.1572-0241.2007.01601.x].

81. M. Coletta et al., "Acetic acid chromoendoscopy for the diagnosis of early neoplasia and specialized intestinal metaplasia in Barrett's esophagus: A meta-analysis," Gastrointest. Endosc. 83(1), 57-67, Elsevier, Inc. (2016) [doi:10.1016/j.gie.2015.07.023].

82. S. Tholoor et al., "Acetic acid chromoendoscopy in Barrett's esophagus surveillance is superior to the standardized random biopsy protocol: results from a large cohort study (with video).," Gastrointest. Endosc. 80(3), 417-424 (2014) [doi:10.1016/j.gie.2014.01.041].

83. J. R. Olliver et al., "Chromoendoscopy with methylene blue and associated DNA damage in Barrett's oesophagus.," Lancet 362(9381), 373-374 (2003).

84. R. G. Sturmey, C. P. Wild, and L. J. Hardie, "Removal of red light minimizes methylene blue-stimulated DNA damage in oesophageal cells: implications for chromoendoscopy.," Mutagenesis 24(3), 253-258 (2009) [doi:10.1093/mutage/gep004].

85. P. Kovacic and R. Somanathan, "Toxicity of imine-iminium dyes and pigments: Electron transfer, radicals, oxidative stress and other physiological effects," J. Appl. Toxicol. 34(8), 825-834 (2014) [doi:10.1002/jat.3005].

86. M. L. James and S. S. Gambhir, "A Molecular Imaging Primer: Modalities, Imaging Agents, and Applications," Physiol. Rev. 92(2), 897-965 (2012) [doi:10.1152/physrev.00049.2010].

87. J. H. Lee and T. D. Wang, "Molecular endoscopy for targeted imaging in the digestive tract," Lancet Gastroenterol. Hepatol. 1(2), 147-155, Elsevier Ltd (2016) [doi:10.1016/S2468-1253(16)30027-9].

88. M. B. Sturm et al., "Targeted Imaging of Esophageal Neoplasia with a Fluorescently Labeled Peptide: First-inHuman Results," Sci. Transl. Med. 5(184), 184ra61 (2013) [doi:10.1126/scitranslmed.3004733.Targeted].

89. B. P. Joshi et al., "Multimodal endoscope can quantify wide-field fluorescence detection of Barrett's neoplasia.," Endoscopy (2015) [doi:10.1055/s-0034-1392803].

90. E. L. Bird-Lieberman et al., "Molecular imaging using fluorescent lectins permits rapid endoscopic identification of dysplasia in Barrett's esophagus.," Nat. Med. 18(2), 315-321 (2012) [doi:10.1038/nm.2616].

91. W. B. Nagengast et al., "Near-infrared fluorescence molecular endoscopy detects dysplastic oesophageal lesions using topical and systemic tracer of vascular endothelial growth factor A," Gut, gutjnl-2017-314953 (2017) [doi:10.1136/gutjnl-2017-314953].

92. K. Kaneko et al., "Effect of novel bright image enhanced endoscopy using blue laser imaging (BLI)," Endosc. Int. Open 02(04), E212-E219 (2014) [doi:10.1055/s-0034-1390707].

93. N. Yoshida et al., "Ability of a novel blue laser imaging system for the diagnosis of colorectal polyps," Dig. Endosc. 26(2), 250-258 (2014) [doi:10.1111/den.12127].

94. Y. Miyake et al., "Development of New Electronic Endoscopes Using the Spectral Images of an Internal Organ," in Proceedings of the IS\&T/SID's Thirteen Color Imaging Conference, pp. 261-269, Society for Imaging Science and Technology (2005).

95. S. Kodashima and M. Fujishiro, "Novel image-enhanced endoscopy with i-scan technology," World J. Gastroenterol. 16(9), 1043-1049 (2010) [doi:10.3748/wjg.v16.i9.1043].

96. C. Q. Li et al., "Magnified and enhanced computed virtual chromoendoscopy in gastric neoplasia: A feasibility study," World J. Gastroenterol. 19(26), 4221-4227 (2013) [doi:10.3748/wjg.v19.i26.4221].

97. H. Osawa et al., "Diagnosis of depressed-type early gastric cancer using small-caliber endoscopy with flexible spectral imaging color enhancement," Dig. Endosc. 24(4), 231-236 (2012) [doi:10.1111/j.1443- 
1661.2011.01224.x].

98. H. Osawa et al., "Diagnosis of endoscopic Barrett's esophagus by transnasal flexible spectral imaging color enhancement," J. Gastroenterol. 44(11), 1125-1132 (2009) [doi:10.1007/s00535-009-0121-z].

99. A. Aminalai et al., "Live image processing does not increase adenoma detection rate during colonoscopy: a randomized comparison between FICE and conventional imaging (Berlin Colonoscopy Project 5, BECOP-5).," Am. J. Gastroenterol. 105(11), 2383-2388 (2010) [doi:10.1038/ajg.2010.273].

100. S. J. Chung et al., "Efficacy of computed virtual chromoendoscopy on colorectal cancer screening: a prospective, randomized, back-to-back trial of Fuji Intelligent Color Enhancement versus conventional colonoscopy to compare adenoma miss rates," Gastrointest. Endosc. 72(1), 136-142, Elsevier Inc. (2010) [doi:10.1016/j.gie.2010.01.055].

101. P. J. Basford et al., "High-definition endoscopy with i-Scan for evaluation of small colon polyps: The HiSCOPE study," Gastrointest. Endosc. 79(1), 111-118, Elsevier Ltd (2014) [doi:10.1016/j.gie.2013.06.013].

102. S. N. Hong et al., "Prospective, randomized, back-to-back trial evaluating the usefulness of i-SCAN in screening colonoscopy," Gastrointest. Endosc. 75(5), 1011-1021.e2, Elsevier Inc. (2012) [doi:10.1016/j.gie.2011.11.040].

103. C. K. Lee, S. H. Lee, and Y. Hwangbo, "Narrow-band imaging versus I-Scan for the real-time histological prediction of diminutive colonic polyps: A prospective comparative study by using the simple unified endoscopic classification," Gastrointest. Endosc. 74(3), 603-609, Elsevier Inc. (2011) [doi:10.1016/j.gie.2011.04.049].

104. Y. Yoshida et al., "A randomized crossover open trial of the adenoma miss rate for narrow band imaging (NBI) versus flexible spectral imaging color enhancement (FICE)," Int. J. Colorectal Dis. 28(11), 1511-1516 (2013) [doi:10.1007/s00384-013-1735-4].

105. S. J. Chung et al., "Comparison of detection and miss rates of narrow band imaging, flexible spectral imaging chromoendoscopy and white light at screening colonoscopy: a randomised controlled back-to-back study," Gut 63(5), 785-791 (2014) [doi:10.1136/gutjnl-2013-304578].

106. E. Masci et al., "Interobserver agreement among endoscopists on evaluation of polypoid colorectal lesions visualized with the Pentax i-Scan technique," Dig. Liver Dis. 45(3), 207-210 (2013) [doi:10.1016/j.dld.2012.09.012].

107. F. Pigò et al., "I-Scan high-definition white light endoscopy and colorectal polyps: Prediction of histology, interobserver and intraobserver agreement," Int. J. Colorectal Dis. 28(3), 399-406 (2013) [doi:10.1007/s00384012-1583-7].

108. P. Sharma et al., "Development and Validation of a Classification System to Identify High-grade Dysplasia and Esophageal Adenocarcinoma in Barrett's Esophagus Using Narrow Band Imaging.," Gastroenterology 150(3), 591-598, Elsevier, Inc (2016) [doi:10.1053/j.gastro.2015.11.037].

109. J. Haringsma, M. Gastroenterologist, and G. N. J. Tytgat, "Fluorescence and autofluorescence," Baillieres. Clin. Gastroenterol. 13(1), 1-10 (1999) [doi:10.1053/bega.1999.0003].

110. M. A. Kara et al., "Endoscopic video autofluorescence imaging may improve the detection of early neoplasia in patients with Barrett's esophagus," Gastrointest. Endosc. 61(6), 679-685 (2005) [doi:10.1016/S00165107(04)02577-5].

111. W. L. Curvers et al., "Endoscopic trimodal imaging versus standard video endoscopy for detection of early Barrett's neoplasia: a multicenter, randomized, crossover study in general practice.," Gastrointest. Endosc. 73(2), 195-203 (2011) [doi:10.1016/j.gie.2010.10.014].

112. D. F. Boerwinkel et al., "Endoscopic TriModal imaging and biomarkers for neoplasia conjoined: a feasibility study in Barrett's esophagus.," Dis. Esophagus 27(5), 435-443 (2014) [doi:10.1111/j.1442-2050.2012.01428.x].

113. W. L. Curvers et al., "Endoscopic tri-modal imaging is more effective than standard endoscopy in identifying early-stage neoplasia in Barrett's esophagus.," Gastroenterology 139(4), 1106-1114, Elsevier Inc. (2010) [doi:10.1053/j.gastro.2010.06.045].

114. M. Wallace et al., "Miami classification for probe-based confocal laser endomicroscopy.," Endoscopy 43(10), 882-891, (C) Georg Thieme Verlag KG (2011) [doi:10.1055/s-0030-1256632].

115. R. Kiesslich et al., "In vivo histology of Barrett's esophagus and associated neoplasia by confocal laser endomicroscopy.," Clin. Gastroenterol. Hepatol. 4(8), 979-987 (2006) [doi:10.1016/j.cgh.2006.05.010].

116. B. J. Vakoc et al., "Comprehensive esophageal microscopy by using optical frequency-domain imaging (with video)\{A figure is presented\}," Gastrointest. Endosc. 65(6), 898-905 (2007) [doi:10.1016/j.gie.2006.08.009].

117. M. J. Gora et al., "Tethered capsule endomicroscopy enables less invasive imaging of gastrointestinal tract microstructure.," Nat. Med. 19(2), 238-240, Nature Publishing Group (2013) [doi:10.1038/nm.3052].

118. S. H. Yun et al., "Comprehensive volumetric optical microscopy in vivo.," Nat. Med. 12(12), 1429-1433 (2006) [doi:10.1038/nm1450].

119. H. C. Wolfsen et al., "Safety and feasibility of volumetric laser endomicroscopy in patients with Barrett's esophagus (with videos)," Gastrointest. Endosc. 82(4), 631-640, Elsevier, Inc. (2015) 
[doi:10.1016/j.gie.2015.03.1968].

120. A. F. Swager et al., "Detection of buried Barrett's glands after radiofrequency ablation with volumetric laser endomicroscopy," Gastrointest. Endosc. 83(1), 80-88, Elsevier, Inc. (2016) [doi:10.1016/j.gie.2015.05.028].

121. A. Swager et al., "Volumetric laser endomicroscopy in Barrett's esophagus: A feasibility study on histological correlation," Dis. Esophagus, 1-8 (2015) [doi:10.1111/dote.12371].

122. A. J. Trindade et al., "Volumetric laser endomicroscopy can target neoplasia not detected by conventional endoscopic measures in long segment Barrett's esophagus.," Endosc. Int. open 4(3), E318-22 (2016) [doi:10.1055/s-0042-101409].

123. C. L. Leggett et al., "Comparative diagnostic performance of volumetric laser endomicroscopy and confocal laser endomicroscopy in the detection of dysplasia associated with Barrett's esophagus," Gastrointest. Endosc. 83(5), 880-888.e2, Elsevier, Inc. (2015) [doi:10.1016/j.gie.2015.08.050].

124. J. M. Poneros et al., "Diagnosis of specialized intestinal metaplasia by optical coherence tomography.," Gastroenterology 120(1), 7-12 (2001).

125. J. A. Evans et al., "Optical coherence tomography to identify intramucosal carcinoma and high-grade dysplasia in Barrett's esophagus.," Clin. Gastroenterol. Hepatol. 4(1), 38-43 (2006).

126. J. A. Evans et al., "Identifying intestinal metaplasia at the squamocolumnar junction by using optical coherence tomography," 50-56 (2007) [doi:10.1016/j.gie.2006.04.027.Identifying].

127. J. Sauk et al., "Interobserver agreement for the detection of barrett's esophagus with optical frequency domain imaging," Dig. Dis. Sci. 58(8), 2261-2265 (2013) [doi:10.1007/s10620-013-2625-x].

128. M. J. Suter et al., "Esophageal-guided biopsy with volumetric laser endomicroscopy and laser cautery marking: $A$ pilot clinical study," Gastrointest. Endosc. 79(6), 886-896, Elsevier (2014) [doi:10.1016/j.gie.2013.11.016].

129. M. J. Gora et al., "Imaging the upper gastrointestinal tract in unsedated patients using tethered capsule endomicroscopy," Gastroenterology 145(4), 723-725, Elsevier, Inc (2013) [doi:10.1053/j.gastro.2013.07.053].

130. G. J. Ughi et al., "Automated segmentation and characterization of esophageal wall in vivo by tethered capsule optical coherence tomography endomicroscopy," 660-665 (2016) [doi:10.1364/BOE.7.000409].

131. M. Gora et al., "Tethered capsule endomicroscopy : from bench to bedside at a primary care practice Tethered capsule endomicroscopy : from bench to bedside at a primary care practice," J. Biomed. Opt. 21(10), 104001 (2016) [doi:10.1117/1.JBO.21.10.104001.Downloaded].

132. "ESOTRAC,"<https://www.esotrac2020.eu/> (accessed 27 March 2018).

133. M. S. Bergholt et al., "Fiberoptic confocal raman spectroscopy for real-time in vivo diagnosis of dysplasia in Barrett's esophagus," Gastroenterology 146(1), 27-32 (2014) [doi:10.1053/j.gastro.2013.11.002].

134. L. M. Almond et al., "Endoscopic Raman spectroscopy enables objective diagnosis of dysplasia in Barrett's esophagus," Gastrointest. Endosc. 79(1), 37-45, Elsevier Ltd (2014) [doi:10.1016/j.gie.2013.05.028].

135. G. A. Van Norman, "Drugs and Devices: Comparison of European and U.S. Approval Processes," JACC Basic to Transl. Sci. 1(5), 399-412, Elsevier (2016) [doi:10.1016/j.jacbts.2016.06.003].

136. D. M. Beswick, A. Kaushik, and D. Beinart, "Biomedical device innovation methodology: applications in biophotonics," J. Biomed. Opt. 23(02), 1 (2017) [doi:10.1117/1.JBO.23.2.021102].

137. M. Laudicella et al., "Cost of care for cancer patients in England: Evidence from population-based patient-level data," Br. J. Cancer 114(11), 1286-1292, Nature Publishing Group (2016) [doi:10.1038/bjc.2016.77].

138. P. J. Trivedi and B. Braden, "Indications, stains and techniques in chromoendoscopy.," QJM 106(2), 117-131 (2013) [doi:10.1093/qjmed/hcs186].

139. E. M. Sevick-Muraca et al., "Advancing the translation of optical imaging agents for clinical imaging.," Biomed. Opt. Express 4(1), 160-170 (2013) [doi:10.1364/BOE.4.000160].

140. W. S. Tummers et al., "Regulatory aspects of optical methods and exogenous targets for cancer detection," Cancer Res. 77(9), 2197-2206 (2017) [doi:10.1158/0008-5472.CAN-16-3217].

141. "Overview of Device Regulation,"

<https://www.fda.gov/MedicalDevices/DeviceRegulationandGuidance/Overview/default.htm> (accessed 27 November 2017).

142. "Medical devices - European Commission," <https://ec.europa.eu/growth/sectors/medical-devices_en> (accessed 27 November 2017).

143. L. Marcu et al., "Biophotonics: the big picture," J. Biomed. Opt. 23(02), 1 (2017) [doi:10.1117/1.JBO.23.2.021103].

144. D. F. Boerwinkel et al., "Third-generation autofluorescence endoscopy for the detection of early neoplasia in Barrett's esophagus: a pilot study.," Dis. Esophagus 27(3), 276-284 (2014) [doi:10.1111/dote.12094]. 
145. L. Alvarez Herrero et al., "Zooming in on Barrett oesophagus using narrow-band imaging: an international observer agreement study.," Eur. J. Gastroenterol. Hepatol. 21(9), 1068-1075 (2009) [doi:10.1097/MEG.0b013e3283271e87].

146. W. L. Curvers et al., "Mucosal morphology in Barrett's esophagus: interobserver agreement and role of narrow band imaging.," Endoscopy 40(10), 799-805, ( Georg Thieme Verlag KG Stuttgart · New York (2008) [doi:10.1055/s-2008-1077596].

147. F. B. Silva et al., "Endoscopic assessment and grading of Barrett's esophagus using magnification endoscopy and narrow-band imaging: Accuracy and interobserver agreement of different classification systems (with videos)," Gastrointest. Endosc. 73(1), 7-14, Elsevier Inc. (2011) [doi:10.1016/j.gie.2010.09.023].

148. M. a Kara et al., "Detection and classification of the mucosal and vascular patterns (mucosal morphology) in Barrett's esophagus by using narrow band imaging.," Gastrointest. Endosc. 64(2), 155-166 (2006) [doi:10.1016/j.gie.2005.11.049].

149. R. Singh et al., "Narrow-band imaging with magnification in Barrett's esophagus: validation of a simplified grading system of mucosal morphology patterns against histology.," Endoscopy 40(6), 457-463 (2008) [doi:10.1055/s-2007-995741].

150. P. Sharma et al., "The utility of a novel narrow band imaging endoscopy system in patients with Barrett's esophagus," Gastrointest. Endosc. 64(2), 167-175 (2006) [doi:10.1016/j.gie.2005.10.044].

151. M. de Bruijne, "Machine learning approaches in medical image analysis: From detection to diagnosis," Med. Image Anal. 33, 94-97, Elsevier B.V. (2016) [doi:10.1016/j.media.2016.06.032].

152. K. Suzuki, "Overview of deep learning in medical imaging," Radiol. Phys. Technol. 10(3), 1-17, Springer Singapore (2017) [doi:10.1007/s12194-017-0406-5].

153. P. B. Garcia-Allende et al., "Towards clinically translatable NIR fluorescence molecular guidance for colonoscopy.," Biomed. Opt. Express 5(1), 78-92 (2013) [doi:10.1364/BOE.5.000078].

154. P. E. Pelargos et al., "Utilizing virtual and augmented reality for educational and clinical enhancements in neurosurgery," J. Clin. Neurosci. 35((in press)), 1-4, Elsevier Ltd (2016) [doi:10.1016/j.jocn.2016.09.002]

155. S. Nicolau et al., "Augmented reality in laparoscopic surgical oncology," Surg. Oncol. 20(3), 189-201, Elsevier Ltd (2011) [doi:10.1016/j.suronc.2011.07.002].

156. M. A. Manfredi et al., "Electronic chromoendoscopy," Gastrointest. Endosc. 81(2), 249-261 (2015) [doi:10.1016/j.gie.2014.06.020].

157. K. Kandiah et al., "OC-054 Development and Validation of a Classification System to Identify Barrett's Neoplasia Using Acetic Acid Chromoendoscopy: The Predict Classification: Abstract OC-054 Table 1," Gut 65(Suppl 1), A31.1-A31, BMJ Publishing Group (2016) [doi:10.1136/gutjnl-2016-312388.53].

158. G. Longcroft-Wheaton et al., "Duration of acetowhitening as a novel objective tool for diagnosing high risk neoplasia in Barrett's esophagus: A prospective cohort trial," Endoscopy 45(6), 426-432 (2013) [doi:10.1055/s0032-1326630].

159. B. C. Wilson, M. Jermyn, and F. Leblond, "Challenges and opportunities in clinical translation of biomedical optical spectroscopy and imaging," J. Biomed. Opt. 23(03), 1 (2018) [doi:10.1117/1.JBO.23.3.030901].

160. ICNIRP, "ICNIRP guidelines on limits of exposure to incoherent visible and infrared radiation," Health Phys. 71(5), 804-819 (2013) [doi:10.1097/HP.0b013e3182983fd4].

161. C. K. Brookner et al., "Safety Analysis: Relative Risks of Ultraviolet Exposure from Fluorescence Spectroscopy and Colposcopy Are Comparable," Photochem. Photobiol. 65(6), 1020-1025 (1997) [doi:10.1111/j.17511097.1997.tb07963.x].

162. “DIRECTIVE 2006/25/EC OF THE EUROPEAN PARLIAMENT AND OF THE COUNCIL” (2006).

163. NEMA, "Performance Measurements of Positron Emission Tomographs (PETs)," 2013, <https://www.nema.org/Standards/Pages/Performance-Measurements-of-Positron-Emission-Tomographs.aspx>.

164. R. Boellaard et al., "FDG PET/CT: EANM procedure guidelines for tumour imaging: version 2.0," Eur. J. Nucl. Med. Mol. Imaging 42(2), 328-354 (2014) [doi:10.1007/s00259-014-2961-x].

165. B. Zhu et al., "Determining the Performance of Fluorescence Molecular Imaging Devices using Traceable Working Standards with SI Units of Radiance," IEEE Trans. Med. Imaging 0062(c), 1-1 (2015) [doi:10.1109/TMI.2015.2496898].

166. E. Downs-Kelly et al., "Poor interobserver agreement in the distinction of high-grade dysplasia and adenocarcinoma in pretreatment Barrett's esophagus biopsies.," Am. J. Gastroenterol. 103(9), 2333-40; quiz 2341 (2008) [doi:10.1111/j.1572-0241.2008.02020.x].

167. J. P. B. O'Connor et al., "Imaging intratumor heterogeneity: Role in therapy response, resistance, and clinical outcome," Clin. Cancer Res. 21(2), 249-257 (2015) [doi:10.1158/1078-0432.CCR-14-0990].

168. J. A. Usher-Smith, S. J. Sharp, and S. J. Griffin, "The spectrum effect in tests for risk prediction, screening, and 
diagnosis," BMJ, i3139 (2016) [doi:10.1136/bmj.i3139].

169. M. A. Calin et al., "Hyperspectral Imaging in the Medical Field: Present and Future," Appl. Spectrosc. Rev. 49(6), 435-447 (2014) [doi:10.1080/05704928.2013.838678].

170. D. Grosenick et al., "Review of optical breast imaging and spectroscopy," J. Biomed. Opt. 21(9), 091311 (2016) [doi:10.1117/1.JBO.21.9.091311].

171. C. Schäfauer, S. Clade, and F. Lacombe, "Surgical applications of probe based confocal endomicroscopy: the benefit of immaterial biopsies," in Proceedings of SPIE, p. 217 (2016).

172. A. P. Kassianos et al., "Smartphone applications for melanoma detection by community, patient and generalist clinician users: A review,” Br. J. Dermatol. 172(6), 1507-1518 (2015) [doi:10.1111/bjd.13665].

173. N. Farahani et al., "International Telepathology: Promises and Pitfalls," Pathobiology 83(2-3), 121-126 (2016) [doi:10.1159/000442390].

174. E. Tensen et al., "Two Decades of Teledermatology: Current Status and Integration in National Healthcare Systems," Curr. Dermatol. Rep. 5(2), 96-104 (2016) [doi:10.1007/s13671-016-0136-7].

175. J. D. Cohen et al., "Detection and localization of surgically resectable cancers with a multi-analyte blood test," 926-930 (2018) [doi:10.1126/science.aar3247].

176. C. Chi et al., "Intraoperative imaging-guided cancer surgery: From current fluorescence molecular imaging methods to future multi-modality imaging technology," Theranostics 4(11), 1072-1084 (2014) [doi:10.7150/thno.9899].

177. E. De Boer et al., "Optical innovations in surgery," Br. J. Surg. 102(2), 56-72 (2015) [doi:10.1002/bjs.9713].

178. K. Heinzmann et al., "Multiplexed imaging for diagnosis and therapy," Nat. Biomed. Eng. 1(9), 697-713, Springer US (2017) [doi:10.1038/s41551-017-0131-8].

179. Y. M. Bhat et al., "High-definition and high-magnification endoscopes," Gastrointest. Endosc. 80(6), 919-927 (2014) [doi:10.1016/j.gie.2014.06.019].

180. "Olympus -Technologies NBI| Medical Systems," <https://www.olympus-

europa.com/medical/en/medical_systems/technologies/narrow_band_imaging_nbi_1/technologies_nbi.jsp> (accessed 26 April 2017).

181. "FICE Dual Mode | Fujifilm Europe," <https://www.fujifilm.eu/eu/products/medicalsystems/endoscopy/technology/fice-dual-mode> (accessed 26 April 2017).

182. "Advanced Imaging | PENTAX Medical (EMEA)," <https://www.pentaxmedical.com/pentax/en/95/1/i-scanimaging> (accessed 26 April 2017).

183. "MedX Health - About SIMSYS-MoleMate ${ }^{\mathrm{TM}}$," $<$ http://medxhealth.com/OurProducts/SIAscopytrade;/overview.aspx> (accessed 26 April 2017).

184. "MelaFind," <http://www.melafind.com/melafind/> (accessed 26 April 2017).

185. "Spectral Molecular Imaging - Products," <http://www.opmol.com/products.html> (accessed 26 April 2017).

186. R. Rameshshanker and A. Wilson, "Electronic Imaging in Colonoscopy: Clinical Applications and Future Prospects," Curr. Treat. Options Gastroenterol. 14(1), 140-151 (2016) [doi:10.1007/s11938-016-0075-1].

187. G. Lu and B. Fei, "Medical hyperspectral imaging: a review.," J. Biomed. Opt. 19(1), 10901 (2014) [doi:10.1117/1.JBO.19.1.010901].

188. J. Jang, "The Past, Present, and Future of Image-Enhanced Endoscopy," 466-475 (2015).

189. "[DERMA MEDICAL SYSTEMS] English," <https://www.dermamedicalsystems.com/index.php?menu_id=117> (accessed 2 May 2017).

190. S. Alali and A. Vitkin, "Polarized light imaging in biomedicine: emerging Mueller matrix methodologies for bulk tissue assessment.," J. Biomed. Opt. 20(6), 61104 (2015) [doi:10.1117/1.JBO.20.6.061104].

191. "Cellvizio: Our Flagship Product | Mauna Kea Technologies," <http://www.maunakeatech.com/en/hospitaladministrators/cellvizio-solution> (accessed 26 April 2017).

192. "ViewnVivo Home - ViewnVivo - Must-see, Miniaturised In Vivo Microscopy," <http://viewnvivo.com/> (accessed 26 April 2017).

193. "Caliber I.D. - Clinical Applications," <http://www.caliberid.com/clinical.html> (accessed 2 May 2017).

194. "Olympus - GIF-FQ260Z | Medical Systems,"

<https://www.olympus.co.uk/medical/en/medical_systems/products_services/product_details/product_details_90 27.jsp> (accessed 26 April 2017).

195. "Olympus - CF-FH260AZL/I | Medical Systems,"

$<$ https://www.olympus.co.uk/medical/en/medical_systems/products_services/product_details/product_details_91 55.jsp> (accessed 26 April 2017). 
196. "BF-F260 | Endoscopes | All Products | Products | Olympus Medical Hong Kong,"

$<$ http://olympusmedical.com.hk/products/all-products/endoscopes/bronchoscopes/videoscopes/bff260/index.html> (accessed 26 April 2017).

197. L. M. Wong Kee Song et al., "Autofluorescence imaging," Gastrointest. Endosc. 73(4), 647-650 (2011) [doi:10.1016/j.gie.2010.11.006].

198. “Jenlab: Dermalnspect," <http://www.jenlab.de/Dermalnspect.29.0.html> (accessed 5 May 2017).

199. K. M. Hanson and C. J. Bardeen, "Application of nonlinear optical microscopy for imaging skin," Photochem. Photobiol. 85(1), 33-44 (2009) [doi:10.1111/j.1751-1097.2008.00508.x].

200. L. Fu and M. Gu, "Fibre-optic nonlinear optical microscopy and endoscopy," J. Microsc. 226(3), 195-206 (2007) [doi:10.1111/j.1365-2818.2007.01777.x].

201. G. Thomas et al., "Advances and challenges in label-free nonlinear optical imaging using two-photon excitation fluorescence and second harmonic generation for cancer research," J. Photochem. Photobiol. B Biol. 141, 128138, Elsevier B.V. (2014) [doi:10.1016/j.jphotobiol.2014.08.025].

202. "NinePoint Medical," <http://www.ninepointmedical.com/\#NvisionVLE> (accessed 26 April 2017).

203. "Verisante Technology, Inc.," <http://www.verisante.com/products/core/> (accessed 26 April 2017).

204. "Product - Vivosight," <https://vivosight.com/about-us/product/> (accessed 28 April 2017).

205. A. J. Trindade, M. S. Smith, and D. K. Pleskow, "The new kid on the block for advanced imaging in Barrett's esophagus: a review of volumetric laser endomicroscopy.," Therap. Adv. Gastroenterol. 9(3), 408-416 (2016) [doi:10.1177/1756283X16639003].

206. "Verisante AuraTM," <http://www.verisante.com/aura/medical_professional/> (accessed 26 April 2017).

207. C. Kallaway et al., "Advances in the clinical application of Raman spectroscopy for cancer diagnostics," Photodiagnosis Photodyn. Ther. 10(3), 207-219, Elsevier B.V. (2013) [doi:10.1016/j.pdpdt.2013.01.008].

208. W. Wang et al., "Real-time in vivo cancer diagnosis using raman spectroscopy," J. Biophotonics 8(7), 527-545 (2015) [doi:10.1002/jbio.201400026].

209. Q. Tu and C. Chang, "Diagnostic applications of Raman spectroscopy," Nanomedicine Nanotechnology, Biol. Med. 8(5), 545-558, Elsevier Inc. (2012) [doi:10.1016/j.nano.2011.09.013].

210. "Advanced Imaging | PENTAX Medical (EMEA)," <https://www.pentaxmedical.com/pentax/en/95/1/WavSTAT4Optical-Biopsy-System> (accessed 26 April 2017).

211. Z. Benes and Z. Antos, "Optical biopsy system distinguishing between hyperplastic and adenomatous polyps in the colon during colonoscopy," Anticancer Res. 29(11), 4737-4739 (2009).

212. D. F. Boerwinkel et al., "Fluorescence spectroscopy incorporated in an Optical Biopsy System for the detection of early neoplasia in Barrett's esophagus.," Dis. Esophagus (2014) [doi:10.1111/dote.12193].

213. G. Yu, "Near-infrared diffuse correlation spectroscopy in cancer diagnosis and therapy monitoring," J. Biomed. Opt. 17(1), 010901 (2012) [doi:10.1117/1.JBO.17.1.010901]. 